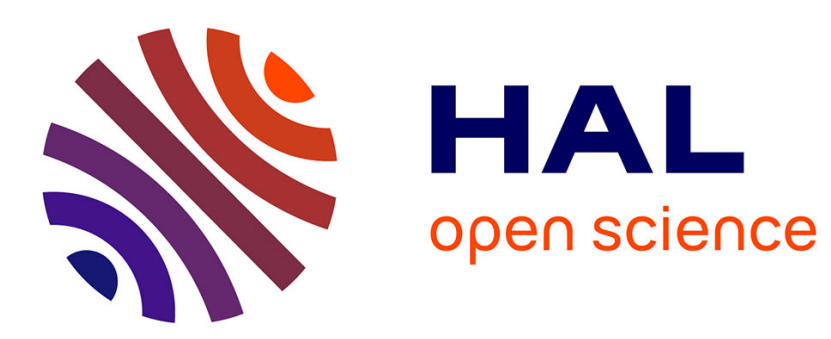

\title{
Continuous Mesh Framework Part I: Well-Posed Continuous Interpolation Error
}

\author{
Adrien Loseille, Frédéric Alauzet
}

\section{To cite this version:}

Adrien Loseille, Frédéric Alauzet. Continuous Mesh Framework Part I: Well-Posed Continuous Interpolation Error. SIAM Journal on Numerical Analysis, 2011, 49 (1), pp.38-60. 10.1137/090754078 . hal-03167249

\section{HAL Id: hal-03167249 \\ https://hal.science/hal-03167249}

Submitted on 15 Mar 2021

HAL is a multi-disciplinary open access archive for the deposit and dissemination of scientific research documents, whether they are published or not. The documents may come from teaching and research institutions in France or abroad, or from public or private research centers.
L'archive ouverte pluridisciplinaire HAL, est destinée au dépôt et à la diffusion de documents scientifiques de niveau recherche, publiés ou non, émanant des établissements d'enseignement et de recherche français ou étrangers, des laboratoires publics ou privés. 


\title{
CONTINUOUS MESH FRAMEWORK PART I: WELL-POSED CONTINUOUS INTERPOLATION ERROR
}

\author{
ADRIEN LOSEILLE* AND FRÉDÉRIC ALAUZET ${ }^{\dagger}$
}

\begin{abstract}
In the context of mesh adaptation, Riemannian metric spaces have been used to prescribe orientation, density and stretching of anisotropic meshes. But, such structures are only considered to compute lengths in adaptive mesh generators. In this article, a Riemannian metric space is shown to be more than a way to compute a length. It is proven to be a reliable continuous mesh model. In particular, we demonstrate that the linear interpolation error can be evaluated continuously on a Riemannian metric space.

From one hand, this new continuous framework proves that prescribing a Riemannian metric field is equivalent to the local control in $\mathbf{L}^{1}$ norm of the interpolation error. This proves the consistency of classical metric-based mesh adaptation procedures. On the other hand, powerful mathematical tools are available and well defined on Riemannian metric spaces: calculus of variations, differentiation, optimization, .... whereas these tools are not defined on discrete meshes.
\end{abstract}

Key words. Unstructured mesh, continuous mesh, Riemannian metric space, interpolation error, linear interpolate, anisotropic mesh adaptation, optimal interpolation error bound.

AMS subject classifications. 65D05, 65L50, 65N15, 65N50

Introduction. The prescription and the generation of adapted meshes are crucial issues during an adaptive process. There exists a large class of methods to prescribe and to generate adapted meshes depending on the problem at hand along with the mesh specificity: uniform, isotropic, anisotropic, ... The simplest algorithms consist in refining or coarsening the current mesh according to patterns. However, such strategies encounter several bottlenecks. In particular, mesh coarsening is only applied to regions already refined by patterns, i.e., only added patterns can be removed, and they do not allow the generation of anisotropic meshes. Moreover, the quality of the sequence of refined meshes is strongly related to the quality of the initial mesh and this quality can only decrease during the refinement process. A generic and elegant way to prescribe anisotropy is to use the notion of metric and Riemannian metric space. In this framework, an adapted anisotropic mesh is simply the image in the Euclidean space of a uniform mesh in a Riemannian metric space. This approach was initiated in $[6,23,41]$. Distances in the adaptive mesh generator are computed in the Riemannian metric space rather than in the Euclidean one. Practically, it consists in generating a unit mesh where the length of the edges is equal to one with respect to the prescribed Riemannian metric. This method is commonly called metricbased mesh adaptation. There are actually a lot of softwares based on the metric concept. Let us cite Bamg [22] and BL2D [26] in 2D, Yams [19] for discrete surface mesh adaptation and Feflo.a [31], Forge3d [10], Fun3d [25], Gamanic3d [20], MadLib [9], MeshAdap [27], Mmg3d [13], Mom3d [40], Tango [5] and LibAdaptivity [34] in 3D. It is worth mentioning that all these softwares have arisen from different mesh generation methods. The method in $[20,22]$ is based on a global constrained Delaunay kernel. In [26], the Delaunay method and the frontal approaches are coupled. [19] is based on local mesh modifications. And, [10] is based on the minimal volume principle.

As the use of Riemannian metric fields is well-suited for the generation of anisotropic adapted meshes, it is then mandatory to transform the error estimate at hand into

*INRIA Paris-Rocquencourt, Gamma team, France, (Adrien.Loseille@inria.fr).

${ }^{\dagger}$ INRIA Paris-Rocquencourt, Gamma team, France, (Frederic.Alauzet@inria.fr). 
a metric tensor field. When the solution is assumed to be smooth, a straightforward solution consists in controlling the linear interpolation error. As the linear interpolation error involves the Hessian of the solution, a metric-based estimate is easily derived, see $[6,11,17,24]$. When solutions are of low regularity, a priori or a posteriori error estimates need to be modified to come up with a metric tensor field, as in $[16,35]$. However, both approaches generally involve successive bounds to end up with a metric-based estimate. There are neither specific study on the importance of these approximations nor any guaranty of the veracity of the provided upper bound. In this context, we can still wonder if it is a relevant choice to use metric tensor fields for the control of such error estimates. For the linear interpolation error, a lot of numerical examples for real life problems $[5,14,15,17,30,34,36,40]$ tend to answer affirmatively to this question.

In this paper, this question is theoretically addressed and we prove that it is a relevant choice to use metric tensors for anisotropic mesh adaptation. To this end, we propose to push further this idea and to point out that Riemannian metric spaces are more than a way to compute lengths inside adaptive mesh generators.

In the proposed analysis, a strong duality between discrete entities, e.g. elements and meshes, and continuous mathematical objects, e.g. metric tensors and Riemannian metric spaces, is exhibited. Several mathematical results, named geometric invariants, link their respective properties such as orientation, density, etc. Consequently, a new formalism naturally arises from these results. This is the continuous mesh framework where continuous element and continuous mesh models are proposed. This formalism is justified throughout this work.

Next, we demonstrate that this new formalism extends to the study of the linear interpolation error. More precisely, the local interpolation error of a given function on a discrete element can be expressed only as a function of the associated continuous element model. A definition of the continuous linear interpolate with a point-wise expression of the continuous interpolation error can then be deduced. As a result, the continuous interpolation error for a given continuous mesh can be accurately predicted whatever the considered smooth function. Theoretically, the use of a discrete support is no more mandatory to compute the interpolation error.

Overview. The organization of the paper is as follows. In a first section, we recall the differential geometry notions that are repeatedly used throughout this work. Section 2, several results that connect discrete elements and metric tensor on one hand, and discrete meshes and Riemannian metric spaces on the other hand are proved. According to these dualities, continuous element and mesh models are introduced leading to the continuous mesh framework. In the last section, these results are used to rewrite the discrete linear interpolation error in the continuous framework. It leads to a well established notions of continuous linear interpolate and continuous point-wise linear interpolation error.

1. Notion of metric tensor for mesh adaptation. In order to be selfcontained, we recall some notions of differential geometry. It mainly concerns the computation of lengths for different kinds of spaces: the Euclidean space, Euclidean metric spaces and Riemannian metric spaces. We refer to $[2,3,12]$ for a complete review on Riemannian metric spaces.

We use the following notations in the sequel. Bold face symbols, as $\mathbf{a}, \mathbf{b}, \mathbf{u}, \mathbf{v}, \mathbf{x}, \ldots$, denote vectors or points of $\mathbb{R}^{3}$. Vector coordinates are denoted by $\mathbf{x}=\left(x_{1}, x_{2}, x_{3}\right)$. The natural dot product between two vectors $\mathbf{u}$ and $\mathbf{v}$ of $\mathbb{R}^{3}$ is $\langle\mathbf{u}, \mathbf{v}\rangle$. 
1.1. Euclidean metric space. An Euclidean metric space $\left(\mathbb{R}^{3}, \mathcal{M}\right)$ is a finite vector space where the dot product is defined by means of a symmetric definite positive matrix $\mathcal{M}$ such that $\langle\mathbf{u}, \mathbf{v}\rangle_{\mathcal{M}}=\langle\mathbf{u}, \mathcal{M} \mathbf{v}\rangle={ }^{t} \mathbf{u} \mathcal{M} \mathbf{v}$, for $(\mathbf{u}, \mathbf{v}) \in \mathbb{R}^{3} \times \mathbb{R}^{3} . \mathcal{M}$ is written as a $3 \times 3$ matrix verifying:

(i) (symmetric) $\forall(\mathbf{u}, \mathbf{v}) \in \mathbb{R}^{3} \times \mathbb{R}^{3}, \quad\langle\mathbf{u}, \mathcal{M} \mathbf{v}\rangle=\langle\mathbf{v}, \mathcal{M} \mathbf{u}\rangle$

(ii) (positive) $\forall \mathbf{u} \in \mathbb{R}^{3}, \quad\langle\mathbf{u}, \mathcal{M} \mathbf{u}\rangle \geq 0$

(iii) (definite) $\langle\mathbf{u}, \mathcal{M} \mathbf{u}\rangle=0 \Longrightarrow \mathbf{u}=\mathbf{0}$.

These properties ensure that $\mathcal{M}$ defines a dot product. In the following, the matrix $\mathcal{M}$ is simply called a metric tensor or a metric. The simplest example of an Euclidean metric space is given by the identity matrix $I_{3}$ which spans the canonical Euclidean space $\mathbb{R}^{3}$. With the dot product defined by $\mathcal{M}, \mathbb{R}^{3}$ becomes a normed vector space $\left(\mathbb{R}^{3},\|\cdot\|_{\mathcal{M}}\right)$ and a metric vector space $\left(\mathbb{R}^{3}, d_{\mathcal{M}}(.,).\right)$ given the following norm and distance definitions:

- $\forall \mathbf{u} \in \mathbb{R}^{3}, \quad\|\mathbf{u}\|_{\mathcal{M}}=\sqrt{\langle\mathbf{u}, \mathcal{M} \mathbf{u}\rangle}$

- $\forall(\mathbf{u}, \mathbf{v}) \in \mathbb{R}^{3} \times \mathbb{R}^{3}, \quad d_{\mathcal{M}}(\mathbf{u}, \mathbf{v})=\|\mathbf{u}-\mathbf{v}\|_{\mathcal{M}}$.

In these spaces, the length $\ell_{\mathcal{M}}$ of a segment $\mathbf{a b}=[\mathbf{a}, \mathbf{b}]$ is given by the distance between its extremities: $\ell_{\mathcal{M}}(\mathbf{a b})=d_{\mathcal{M}}(\mathbf{a}, \mathbf{b})$. Note that this property is generally wrong for a general Riemannian metric space defined hereafter. In an Euclidean metric space, volumes are still well posed. Given a bounded subset $K$ of $\mathbb{R}^{3}$, the volume of $K$ computed with respect to metric tensor $\mathcal{M}$ is:

$$
|K|_{\mathcal{M}}=\int_{K} \sqrt{\operatorname{det} \mathcal{M}} \mathrm{d} K=\sqrt{\operatorname{det} \mathcal{M}}|K|_{I_{n}} .
$$

Geometric interpretation. We will often refer to the geometric interpretation of a metric tensor. This geometric view plays an important role in the continuous mesh model. In the vicinity $\mathcal{V}(\mathbf{a})$ of point $\mathbf{a}$, the set of points, that are at distance $\varepsilon$ of $\mathbf{a}$, is given by:

$$
\Phi_{\mathcal{M}}(\varepsilon)=\left\{\mathbf{x} \in \mathcal{V}(\mathbf{a}) \mid{ }^{t}(\mathbf{x}-\mathbf{a}) \mathcal{M}(\mathbf{x}-\mathbf{a}) \leq \varepsilon^{2}\right\} .
$$

We note that it is sufficient to describe $\Phi_{\mathcal{M}}(1)$ as $\Phi_{\mathcal{M}}(\varepsilon)$ can be deduced from $\Phi_{\mathcal{M}}(1)$ for all $\varepsilon$ by homogeneity. To describe $\Phi_{\mathcal{M}}(1)$, the spectral decomposition $\mathcal{M}=\mathcal{R} \Lambda{ }^{t} \mathcal{R}$ is used. $\mathcal{R}$ is an orthonormal matrix verifying ${ }^{t} \mathcal{R} \mathcal{R}=\mathcal{R}^{t} \mathcal{R}=I_{3}$. It is composed of the eigenvectors of $\mathcal{M} . \Lambda$ is a diagonal matrix composed of the eigenvalues of $\mathcal{M}$. Eigenvalues $\left(\lambda_{i}\right)_{i=1,3}$ are strictly positive. In the eigenvectors frame, the initial quadratic form ${ }^{t}(\mathbf{x}-\mathbf{a}) \mathcal{M}(\mathbf{x}-\mathbf{a})$ becomes ${ }^{t}(\tilde{\mathbf{x}}-\tilde{\mathbf{a}}) \Lambda(\tilde{\mathbf{x}}-\tilde{\mathbf{a}})$. Consequently, $\Phi_{\mathcal{M}}(1)$ is rewritten in this basis:

$$
\Phi_{\mathcal{M}}(1)=\left\{\tilde{\mathbf{x}} \in \mathcal{V}(\tilde{\mathbf{a}}) \mid \sum_{i=1}^{3}\left(\frac{\tilde{x}_{i}-\tilde{a}_{i}}{h_{i}}\right)^{2} \leq 1\right\} .
$$

The last relation defines an ellipsoid centered at $\mathbf{a}$ with its axes aligned with the principal directions of $\mathcal{M}$. Sizes along these directions are given by $h_{i}=\lambda_{i}^{-\frac{1}{2}}$. We denote by $\mathcal{E}_{\mathcal{M}}$ this ellipsoid. Figure 1.1 depicts $\mathcal{E}_{\mathcal{M}}$. In the sequel, the set $\Phi_{\mathcal{M}}(1)$ is called the unit ball of $\mathcal{M}$ and it is denoted by $\mathcal{B}_{\mathcal{M}}$.

Natural metric mapping. The last information handled by $\mathcal{M}$ is the definition of an application that maps the unit ball $\mathcal{B}_{I_{3}}$ of identity metric $I_{3}$ onto the unit ball $\mathcal{B}_{\mathcal{M}}$ of $\mathcal{M}$. This mapping is given by the application $\mathcal{M}^{-\frac{1}{2}}: \mathbb{R}^{3} \mapsto \mathbb{R}^{3} \cdot \mathcal{M}^{-\frac{1}{2}}$ is defined by the spectral decomposition $\mathcal{M}^{-\frac{1}{2}}=\mathcal{R} \Lambda^{-\frac{1}{2} t} \mathcal{R}$, where $\Lambda^{-\frac{1}{2}}$ is the diagonal matrix 
composed of the inverse of the square root of the eigenvalues of $\mathcal{M}$. This mapping provides another description of the ellipsoid $\mathcal{E}_{\mathcal{M}}$ :

$$
\mathcal{E}_{\mathcal{M}}=\left\{\mathcal{M}^{-\frac{1}{2}} \mathbf{x} \mid\|\mathbf{x}\|_{2}^{2}=1\right\} .
$$
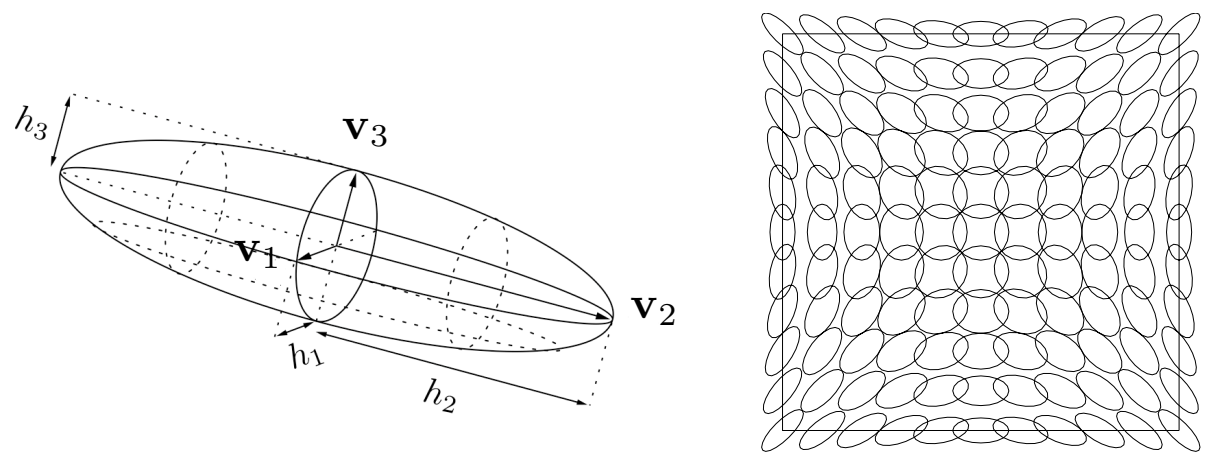

FIG. 1.1. Left, geometric interpretation of $\mathcal{B}_{\mathcal{M}}=\Phi_{\mathcal{M}}(1) . \quad \mathbf{v}_{i}$ are the eigenvectors of $\mathcal{M}$ and $h_{i}^{-2}$ are the eigenvalues of $\mathcal{M}$. Right, geometric visualization of a Riemannian metric space $(\mathcal{M}(\mathbf{x}))_{\mathbf{x} \in[0,1] \times[0,1]}$. At each point $\mathbf{x}$ of the domain, the unit ball of $\mathcal{M}(\mathbf{x})$ is drawn.

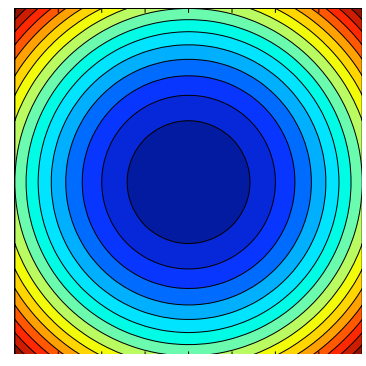

$I_{2}$

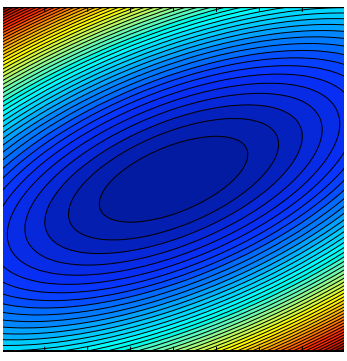

$\mathcal{M}$

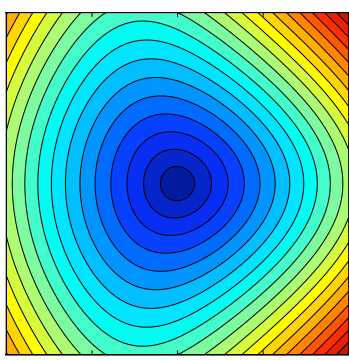

M

FIG. 1.2. Iso-values of the function $f(\mathbf{x})=\ell_{\mathcal{M}}(\mathbf{o x})$ where $\mathbf{o}$ is the origin, i.e., segment length issued from the origin, for different Riemannian metric spaces. Left, in the canonical Euclidean space $\left([-1,1] \times[-1,1], I_{2}\right)$, middle, in an Euclidean metric space $([-1,1] \times[-1,1], \mathcal{M})$ with $\mathcal{M}$ constant and, right, in a Riemannian metric space $(\mathcal{M}(\mathbf{x}))_{\mathbf{x} \in[-1,1]^{2}}$.

1.2. Riemannian metric space. When a metric tensor field is varying smoothly in the whole domain $\Omega$, a Riemannian metric space is defined. We denote this space by $\mathbf{M}=(\mathcal{M}(\mathbf{x}))_{\mathbf{x} \in \Omega}$. The continuous mesh model is based on such a space. To give a practical visualization of a Riemannian metric space, the unit ball of the metric at some points of the domain are drawn, see Figure 1.1 (right).

The main operation performed in this space is the computation of the length of edges. It is important to note that, in a Riemannian metric space, computing the length of a segment (i.e., an edge) differs from evaluating the distance between the extremities of this segment. Indeed, the straight line is no more the shortest path between two points which is given by a geodesic. To take into account the variation of the metric along the edge, the edge length is evaluated with an integral formula:

Definition 1.1 (Edge length computation). In a Riemannian metric space $\mathbf{M}=$ $(\mathcal{M}(\mathbf{x}))_{\mathbf{x} \in \Omega}$, the length of edge $\mathbf{a b}$ is computed using the straight line parameterization 
$\gamma(t)=\mathbf{a}+t \mathbf{a b}$, where $t \in[0,1]:$

$$
\ell_{\mathcal{M}}(\mathbf{a b})=\int_{0}^{1}\left\|\gamma^{\prime}(t)\right\|_{\mathcal{M}} \mathrm{d} t=\int_{0}^{1} \sqrt{{ }^{t} \mathbf{a b} \mathcal{M}(\mathbf{a}+t \mathbf{a b}) \mathbf{a b}} \mathrm{d} t .
$$

Figure 1.2 depicts iso-values of segment length from the origin for different Riemannian metric spaces. The plotted function is $f(\mathbf{x})=\ell_{\mathcal{M}}(\mathbf{o x})$ where $\mathbf{o}$ is the origin of the plane. The iso-values are isotropic for the Euclidean space. They are anisotropic in the case of an Euclidean metric space defined by $\mathcal{M}$. The two principal directions of $\mathcal{M}$ clearly appear. In the case of a Riemannian metric space $\mathbf{M}$, all previous symmetries are lost.

2. Continuous mesh framework. We exemplify in this section the set of meshes that are represented by a Riemannian metric space $\mathbf{M}$. The study is first done locally for an element and then generalized to the whole computational domain $\Omega$. These considerations are based on the concept of a unit element and a unit mesh [18], recalled hereafter. From this analysis, a new duality between discrete and continuous entities will appear clearly.

2.1. Local duality. In this section, $\mathcal{M}$ is a constant metric tensor and $K$ a tetrahedron.

Definition 2.1 (Unit element). An element $K$ is unit with respect to $\mathcal{M}$ if the length of all its edges is unit in the metric $\mathcal{M}$. If $K$ is given by its list of edges $\left(\mathbf{e}_{i}\right)_{i=1,6}$, then :

$$
\forall i=1, \ldots, 6, \quad \ell_{\mathcal{M}}\left(\mathbf{e}_{\mathbf{i}}\right)=1 .
$$

The volume of $K$ is given by:

$$
|K|_{\mathcal{M}}=\frac{\sqrt{2}}{12} \text { and }|K|_{I_{3}}=\frac{\sqrt{2}}{12} \sqrt{\operatorname{det}(\mathcal{M})} .
$$

The relationships between unit discrete elements with respect to $\mathcal{M}$ are stated in the following proposition:

Proposition 2.2 (Equivalence classes). Let $\mathcal{M}$ be a metric tensor, there exists a infinite set of tetrahedra that are unit with respect to $\mathcal{M}$. Conversely, given an element $K=\left(\mathbf{e}_{i}\right)_{i=1,6}$ such that $|K|_{I_{3}} \neq 0$, then there is a unique $\mathcal{M}$ for which element $K$ is unit with respect to $\mathcal{M}$.

The relation unit with respect to $\mathcal{M}$ defines a class of equivalence among the set of all discrete elements.

Proof. We first examine the uniform case where $\mathcal{M}=I_{3}$. The general case is deduced from it by using the mapping $\mathcal{M}^{-\frac{1}{2}}$. Let $K_{0}$ be a regular tetrahedron, thereby $K_{0}$ is unit with respect to $I_{3}$. Whatever the rotation matrix $\mathcal{R}$ verifying ${ }^{t} \mathcal{R} \mathcal{R}=\mathcal{R}^{t} \mathcal{R}=I_{3}$, the tetrahedron $\mathcal{R} K_{0}$ is still unit for $I_{3}$. Consequently, the class of all unit elements for $I_{3}$ is:

$$
\mathcal{K}=\left\{K \mid \forall \mathcal{R} \in \mathcal{O}_{3}: K=\mathcal{R} K_{0}\right\} \text { with } \mathcal{O}_{3}=\left\{\left.\mathcal{R}\right|^{t} \mathcal{R} \mathcal{R}=\mathcal{R}^{t} \mathcal{R}=I_{3}\right\} .
$$

The equivalence class of the unit elements with respect to $\mathcal{M}$ is then given by the set:

$$
\left\{\mathcal{M}^{-\frac{1}{2}} K \mid \forall K \in \mathcal{K}\right\}
$$


Conversely, given a non-degenerated discrete element $K=\left(\mathbf{e}_{i}\right)_{i=1,6}$, such that $|K|_{I_{3}} \neq 0$, let us demonstrate that there exists a unique metric for which $K$ is unit. It is sufficient to solve the following linear system:

$$
(S)\left\{\begin{array}{l}
\ell_{\mathcal{M}}^{2}\left(\mathbf{e}_{\mathbf{1}}\right)=1 \\
\vdots \\
\ell_{\mathcal{M}}^{2}\left(\mathbf{e}_{\mathbf{6}}\right)=1 .
\end{array}\right.
$$

The determinant of $(S)$ is equal to $|K|_{I_{3}} \neq 0$. Consequently, $(S)$ admits a unique solution.

Figure 2.1 depicts some unit elements with respect to a metric tensor (represented geometrically by its unit-ball).
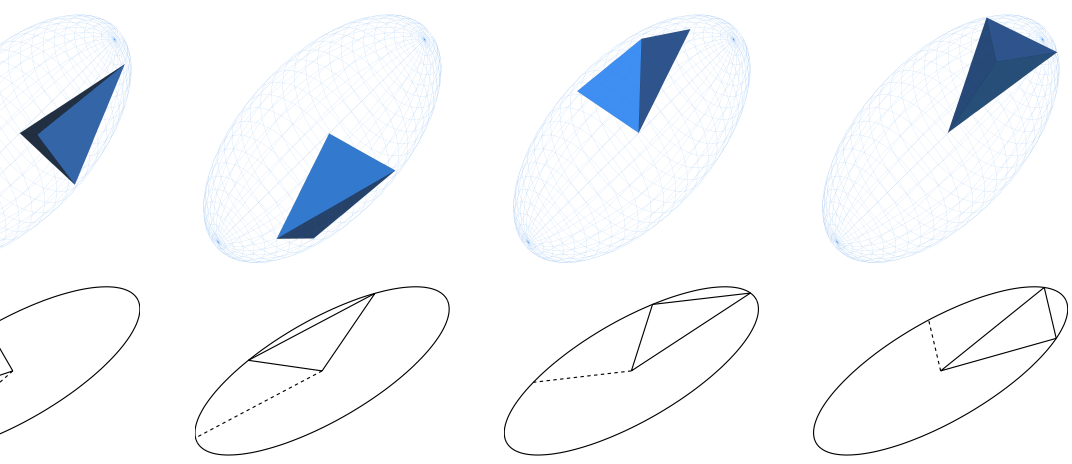

FIG. 2.1. Several unit elements with respect to a continuous element in $2 D$ and $3 D$.
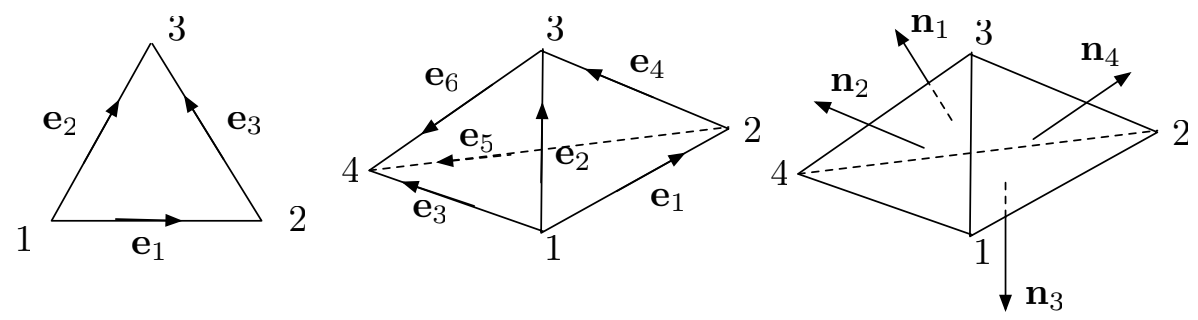

FIG. 2.2. Conventions used to enumerate the edges and the faces of a triangle and of a tetrahedron.

2.2. Geometric invariants. So far, only Definition 2.1 has established relationships between unit elements and the relative metric tensor. Other properties exist. They connect the geometric properties of unit elements to the linear algebra properties of metric tensors. The following proposition gives geometric invariants that hold for all unit elements with respect to a metric tensor.

Proposition 2.3 (Geometric invariants). Let $\mathcal{M}$ be a metric tensor and $K$ be a unit element with respect to $\mathcal{M}$. We denote by $\left(\mathbf{e}_{i}\right)_{i=1,6}$ its edges list, see conventions in Figure 2.2, and by $|K|$ its Euclidean volume. Then, the following invariants hold:

- standard invariants:

$$
\forall\left(\mathbf{e}_{i}, \mathbf{e}_{j}\right), \quad\left\{\begin{array}{l}
{ }^{t} \mathbf{e}_{i} \mathcal{M} \mathbf{e}_{i}=1, \\
2{ }^{t} \mathbf{e}_{i} \mathcal{M} \mathbf{e}_{j}+1=0 \text { if } i \neq j .
\end{array}\right.
$$


- invariant related to the Euclidean volume $|K|$ :

$$
|K|=\frac{\sqrt{3}}{4} \operatorname{det}\left(\mathcal{M}^{-\frac{1}{2}}\right) \text { in } 2 D \text { and }|K|=\frac{\sqrt{2}}{12} \operatorname{det}\left(\mathcal{M}^{-\frac{1}{2}}\right) \text { in } 3 D .
$$

- invariant related to the square length of the edges for all symmetric matrix $H$ :

$$
\begin{aligned}
& \sum_{i=1}^{3}{ }^{t} \mathbf{e}_{i} H \mathbf{e}_{i}=\frac{3}{2} \operatorname{trace}\left(\mathcal{M}^{-\frac{1}{2}} H \mathcal{M}^{-\frac{1}{2}}\right) \text { in } 2 D, \\
& \sum_{i=1}^{6}{ }^{t} \mathbf{e}_{i} H \mathbf{e}_{i}=2 \operatorname{trace}\left(\mathcal{M}^{-\frac{1}{2}} H \mathcal{M}^{-\frac{1}{2}}\right) \text { in } 3 D .
\end{aligned}
$$

Proof. The first invariant of Relation (2.1) stems from the definition of a unit element.

The second invariant of Relation (2.1) states that the angle between two edges of a unit element face is constant in the metric. Let $\left(\mathbf{e}_{i}, \mathbf{e}_{j}\right)$ be a couple of edges of element $K$, this couple defines a face. We denote by $\mathbf{e}_{k}$ the third edge of this face. According to the conventions depicted in Figure 2.2, these edges verify: $\mathbf{e}_{i}+\mathbf{e}_{j}-\mathbf{e}_{k}=\mathbf{0}$. Expanding the following relation

$$
{ }^{t}\left(\mathbf{e}_{i}+\mathbf{e}_{j}+\mathbf{e}_{k}\right) \mathcal{M}\left(\mathbf{e}_{i}+\mathbf{e}_{j}-\mathbf{e}_{k}\right)=0,
$$

leads to the second invariant of Relation (2.1).

Invariant (2.2) is proved by a direct integration. Given a unit element $K$ for $\mathcal{M}$, there exists a unique regular tetrahedron $K_{0}$, which is unit with respect to $I_{3}$, such that $K=\mathcal{M}^{-\frac{1}{2}} K_{0}$. The volume of $K$ is then given by:

$$
|K|=\int_{K} 1 \mathrm{~d} \mathbf{x}=\int_{K_{0}} \operatorname{det}\left(\mathcal{M}^{-\frac{1}{2}}\right) \mathrm{d} \mathbf{x}=\operatorname{det}\left(\mathcal{M}^{-\frac{1}{2}}\right)\left|K_{0}\right|,
$$

where $\left|K_{0}\right|=\frac{\sqrt{2}}{12}$. The same proof applies in $2 \mathrm{D}$.

Invariant (2.3) is first proved in the simpler case where $H=I_{3}$ and $\mathcal{M}=I_{3}$. The general case will be deduced from this proof. Let us consider the regular tetrahedron $K_{0}=\left(\mathbf{x}_{\mathbf{1}}, \mathbf{x}_{\mathbf{2}}, \mathbf{x}_{\mathbf{3}}, \mathbf{x}_{\mathbf{4}}\right)$ unit for $I_{3}$ defined by the list of vertices:

$$
\mathbf{x}_{\mathbf{1}}=(0,0,0), \mathbf{x}_{\mathbf{2}}=(1,0,0), \mathbf{x}_{\mathbf{3}}=\left(\frac{1}{2}, \frac{\sqrt{3}}{2}, 0\right) \text { and } \mathbf{x}_{\mathbf{4}}=\left(\frac{1}{2}, \frac{\sqrt{3}}{6}, \sqrt{\frac{2}{3}}\right) .
$$

The proof does not depend on this specific choice of coordinates. We first demonstrate the following preliminary result: For all lines $(D)$ passing through one of the vertices of $K_{0}$, the sum of the square lengths of the edges projected on $(D)$ is invariant. Without loss of generality, we assume that $(D)$ passes through the vertex $\mathbf{x}_{\mathbf{1}}$ of $K_{0}$. If $(D)$ is defined by the vector

$$
\mathbf{n}=(\cos (u) \cos (v), \cos (u) \sin (v), \sin (u)),
$$

with $(u, v) \in \mathbb{R}^{2}$, then the length of the first three edges of $K_{0}$ projected on $(D)$ are given by:

$$
\begin{aligned}
& a=\mathbf{e}_{\mathbf{1}} \cdot \mathbf{n}=\cos (u) \cos (v), \quad b=\mathbf{e}_{\mathbf{2}} \cdot \mathbf{n}=\frac{1}{2} \cos (u) \cos (v)+\frac{\sqrt{3}}{2} \cos (u) \sin (v), \\
& c=\mathbf{e}_{\mathbf{3}} \cdot \mathbf{n}=\frac{1}{2} \cos (u) \cos (v)+\frac{\sqrt{3}}{6} \cos (u) \sin (v)+\sqrt{\frac{2}{3}} \sin (u),
\end{aligned}
$$


with conventions of Figure 2.2. A direct trigonometric calculus shows that the sum of the square length of all the edges projected on $(D)$ is equal to 2 . Indeed, it comes:

$$
\begin{aligned}
\Sigma & =a^{2}+b^{2}+c^{2}+(b-c)^{2}+(c-a)^{2}+(a-b)^{2} \\
& =3 a^{2}+3 b^{2}+3 c^{2}-2 a b-2 a c-2 b c .
\end{aligned}
$$

After expanding and factorizing, $\Sigma$ reads:

$$
\Sigma=2 \cos (u)^{2} \cos (v)^{2}+2 \cos (u)^{2} \sin (v)^{2}+2 \sin (u)^{2}=2 .
$$

When $\mathcal{M}$ is different from $I_{3}$, we use the mapping $\mathcal{M}^{-\frac{1}{2}}$ that maps the unit ball of $I_{3}$ onto the unit ball of $\mathcal{M}$. As regards line $(D)$, we select the specific line which has for direction vector one of the eigenvectors of $\mathcal{M}$, e.g. $\mathbf{v}_{j}$, and which is passing through $\mathbf{x}_{\mathbf{1}}$. The lengths $a, b$ and $c$ are thus multiplied by $h_{j}$ which is the size prescribed by $\mathcal{M}$ in the direction $\mathbf{v}_{j}$. Consequently, the square length of the edges projected on $(D)$ are multiplied by $h_{j}^{2}$. It comes:

$$
\Sigma_{j}=\sum_{i=1}^{6}\left({ }^{t} \mathbf{e}_{i} \mathbf{v}_{j}\right)^{2}=h_{j}^{2} \Sigma=2 h_{j}^{2} .
$$

Considering the previous relation for all the principal directions of $\mathcal{M}$ and summing the $\Sigma_{j}$ complete the proof:

$$
\sum_{i=1}^{6}\left\|\mathbf{e}_{i}\right\|_{2}^{2}=\sum_{j=1}^{3} \sum_{i=1}^{6}\left({ }^{t} \mathbf{e}_{i} \mathbf{v}_{j}\right)^{2}=2\left(h_{1}^{2}+h_{2}^{2}+h_{3}^{2}\right)=2 \operatorname{trace}\left(\mathcal{M}^{-1}\right) .
$$

We now consider the case where a symmetric matrix $H$ is involved in the estimation. As $H$ is symmetric, it has real eigenvalues $\left(\mu_{i}\right)_{i=1,3}$ along the principal directions $\left(\mathbf{u}_{i}\right)_{i=1,3}$. Let $K$ be a regular tetrahedron given by its list of edges $\left(\mathbf{e}_{i}\right)_{i=1,6}$. According to the preliminary result, the sum of the projected square length of edges $\left(\mathbf{e}_{i}\right)_{i=1,6}$ on each principal direction $\mathbf{u}_{j}$ is equal to 2 . We deduce:

$$
\mu_{j} \sum_{i=1}^{6}\left({ }^{t} \mathbf{e}_{i} \mathbf{u}_{j}\right)^{2}=2 \mu_{j}
$$

for $j \in[1,3]$. Summing the previous equalities lead to:

$$
\sum_{j=1}^{3} \sum_{i=1}^{6} \mu_{j}\left({ }^{t} \mathbf{e}_{i} \mathbf{u}_{j}\right)^{2}=2\left(\mu_{1}+\mu_{2}+\mu_{3}\right) .
$$

The previous equality reads:

$$
\sum_{i=1}^{6}{ }^{t} \mathbf{e}_{i} H \mathbf{e}_{i}=2 \operatorname{trace}(H)
$$

The general case is deduced by taking $\mathcal{M}^{-\frac{1}{2}} H \mathcal{M}^{-\frac{1}{2}}$ as symmetric matrix. In that case, each edge $\mathbf{e}_{i}$ of the regular tetrahedron is mapped on $\tilde{\mathbf{e}}_{i}=\mathcal{M}^{-\frac{1}{2}} \mathbf{e}_{i}$. The new tetrahedron defined by edges $\left(\tilde{\mathbf{e}}_{i}\right)_{i=1,6}$ is unit with respect to $\mathcal{M}$. This concludes the proof.

Other geometric invariants can be found in [28]. 
2.3. Global duality. When dealing with a Riemannian metric space $\mathbf{M}=$ $(\mathcal{M}(\mathbf{x}))_{\mathbf{x} \in \Omega}$, the main complexity is to take into account the variations of the function $\mathbf{x} \mapsto \mathcal{M}(\mathbf{x})$. To simplify the analysis, $\mathbf{M}$ is first rewritten in order to distinguish local properties from global ones.

We recall the spectral decomposition of $\mathcal{M}(\mathbf{x})$ :

$$
\mathbf{M}: \mathbf{x} \in \Omega \mapsto \mathcal{M}(\mathbf{x})=\mathcal{R}(\mathbf{x}) \Lambda(\mathbf{x})^{t} \mathcal{R}(\mathbf{x}),
$$

where diagonal matrix $\Lambda(\mathbf{x})$ is either

$$
\left[\begin{array}{lll}
\lambda_{1}(\mathbf{x}) & & \\
& \lambda_{2}(\mathbf{x}) & \\
& & \lambda_{3}(\mathbf{x})
\end{array}\right] \text { or }\left[\begin{array}{lll}
h_{1}^{-2}(\mathbf{x}) & & \\
& h_{2}^{-2}(\mathbf{x}) & \\
& & h_{3}^{-2}(\mathbf{x})
\end{array}\right] .
$$

$\mathcal{R}(\mathbf{x})$ is an orthonormal matrix providing the local orientation given by the eigenvectors $\left(\mathbf{v}_{i}(\mathbf{x})\right)_{i=1,3},\left(\lambda_{i}(\mathbf{x})\right)_{i=1,3}$ are the local eigenvalues and $\left(h_{i}(\mathbf{x})\right)_{i=1,3}$ are the local sizes along the principal directions of $\mathbf{M}$. Practically, another decomposition is used that points out the local characteristics of $\mathbf{M}$. This decomposition is given by the following proposition.

Proposition 2.4. $\mathbf{M}=(\mathcal{M}(\mathbf{x}))_{\mathbf{x} \in \Omega}$ locally writes:

$$
\mathcal{M}(\mathbf{x})=d^{\frac{2}{3}}(\mathbf{x}) \mathcal{R}(\mathbf{x})\left[\begin{array}{lll}
r_{1}^{-\frac{2}{3}}(\mathbf{x}) & & \\
& r_{2}^{-\frac{2}{3}}(\mathbf{x}) & \\
& & r_{3}^{-\frac{2}{3}}(\mathbf{x})
\end{array}\right]{ }^{t} \mathcal{R}(\mathbf{x}),
$$

where

- the density $d$ is equal to: $d=\left(h_{1} h_{2} h_{3}\right)^{-1}=\left(\lambda_{1} \lambda_{2} \lambda_{3}\right)^{\frac{1}{2}}$,

- the anisotropic quotients $r_{i}$ are equal to: $r_{i}=h_{i}^{3}\left(h_{1} h_{2} h_{3}\right)^{-1}$.

Proof. The proof consists in computing $d^{\frac{2}{3}} r_{i}^{-\frac{2}{3}}$ :

$$
d^{\frac{2}{3}} r_{i}^{-\frac{2}{3}}=\left(\prod_{k=1}^{3} h_{k}\right)^{-\frac{2}{3}} h_{i}^{-2}\left(\prod_{k=1}^{3} h_{k}\right)^{\frac{2}{3}}=h_{i}^{-2}=\lambda_{i} .
$$

The density $d$ controls only the local level of accuracy of $\mathbf{M}$. Increasing or decreasing $d$ does not change the anisotropic properties or the orientation, see Figure 2.3 (left). Anisotropic quotients arises from the quotient of different parallelepipeds, see Figure 2.3 (right).

We also define the complexity $\mathcal{C}$ of $\mathbf{M}$ :

$$
\mathcal{C}(\mathbf{M})=\int_{\Omega} d(\mathbf{x}) d \mathbf{x}=\int_{\Omega} \sqrt{\operatorname{det}(\mathcal{M}(\mathbf{x}))} d \mathbf{x} .
$$

This real-value parameter is useful to quantify the global level of accuracy of $(\mathcal{M}(\mathbf{x}))_{\mathbf{x} \in \boldsymbol{\Omega}}$. It can also be interpreted as the continuous counterpart of the number of vertices of a discrete mesh. This quantity also leads to the definition of sequence of embedded Riemannian spaces. Two embedded Riemannian spaces have the same anisotropic ratios and orientations. They only differ from their complexity:

Definition 2.5 (Embedded Riemannian spaces). Two Riemannian spaces, saying $(\mathcal{M}(\mathbf{x}))_{\mathbf{x} \in \boldsymbol{\Omega}}$ and $(\mathcal{N}(\mathbf{x}))_{\mathbf{x} \in \boldsymbol{\Omega}}$, are embedded if a constant $c$ exists such that:

$$
\forall \mathbf{x} \in \Omega, \quad \mathcal{N}(\mathbf{x})=c \mathcal{M}(\mathbf{x}) .
$$



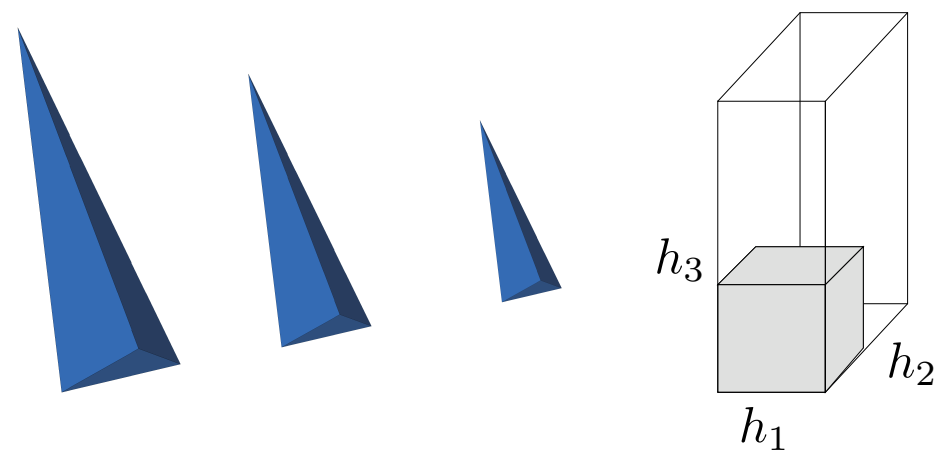

FIG. 2.3. Left, different unit elements where only the density increases from left to right. Right, the geometric interpretation of anisotropic quotients as quotients of parallelepipeds volumes.

Conversely, from $\mathbf{M}=(\mathcal{M}(\mathbf{x}))_{\mathbf{x} \in \boldsymbol{\Omega}}$, we can deduce $\mathbf{N}=(\mathcal{N}(\mathbf{x}))_{\mathbf{x} \in \boldsymbol{\Omega}}$ of complexity $N$ having the same anisotropic properties (anisotropic orientations and ratios) by considering:

$$
\mathcal{N}(\mathbf{x})=\left(\frac{N}{\mathcal{C}(\mathbf{M})}\right)^{\frac{2}{3}} \mathcal{M}(\mathbf{x})
$$

In the context of error estimation, this notion enables convergence order study with respect to an increasing complexity $N$. Consequently, the complexity $\mathcal{C}(\mathbf{M})$ is also the continuous counterpart of the classical parameter $h$ used for uniform meshes while studying convergence. In the continuous framework, the uniform refinement consisting in dividing by two each edge of a uniform mesh of size $h$ writes:

$$
\mathcal{M}_{i}=4^{i}\left[\begin{array}{lll}
\frac{1}{h^{2}} & & \\
& \frac{1}{h^{2}} & \\
& & \frac{1}{h^{2}}
\end{array}\right],
$$

where $i$ is the level of refinement. $\left(\mathcal{M}_{i}\right)_{i=1 \ldots k}$ defines a sequence of embedded Riemannian spaces. Consequently, this simple practical adaptive strategy has a simple continuous interpretation in term of embedded Riemannian spaces. However, when dealing with anisotropic meshes, a unique size $h$ is no more sufficient to give a quantitive information on the accuracy. The size $h$ is then replaced by the complexity.

Unit mesh. The notion of a unit mesh is far more complicated than the notion of a unit element as the existence of a mesh composed only of unit regular simplexes with respect to a given Riemannian space is not guaranteed. For instance, if the Riemannian space is not compatible with the domain size, then it clearly does not exist such discrete mesh. To avoid this problem, let us look at the existence of a discrete mesh composed only with unit regular simplexes with respect to a Riemannian space in $\mathbb{R}^{3}$. To simplify even more the problem, we first consider $\left(I_{3}(\mathbf{x})\right)_{\mathbf{x} \in \mathbb{R}^{3}}$.

It is well known that $\mathbb{R}^{3}$ cannot be filled only with the regular tetrahedron while it is possible to fill $\mathbb{R}^{2}$ with the equilateral triangle. Consequently, even for the simplest case $\left(I_{3}(\mathbf{x})\right)_{\mathbf{x} \in \mathbb{R}^{3}}$, there is no discrete mesh composed only of the unit regular tetrahedron. Therefore, the notion of a unit mesh has to be relaxed:

Definition 2.6 (Unit mesh). A discrete mesh $\mathcal{H}$ of a domain $\Omega \subset \mathbb{R}^{3}$ is unit 
for $(\mathcal{M}(\mathbf{x}))_{\mathbf{x} \in \Omega}$ if all its elements are quasi-unit.

Now, let us give a meaning to quasi-unit in three dimensions. A first way to relax the definition of unity is to take into account technical constraints imposed by mesh generators. To converge (and to avoid cycling) while analyzing the length of edges, the meshing algorithm considers an admissible length interval of the form $\left[\frac{1}{\alpha}, \alpha\right]$ with $\alpha>0$ [19]. If the symmetry property is required, i.e., $\frac{\alpha}{2}=\frac{1}{\alpha}$, then we obtain $\alpha=\sqrt{2}$. Therefore, as regards the meshing requirement, a tetrahedron $K$ defined by its list of edges $\left(\mathbf{e}_{i}\right)_{i=1,6}$ is said quasi-unit if $\forall i \in[1,6], \ell_{\mathcal{M}}\left(\mathbf{e}_{\mathbf{i}}\right) \in\left[\frac{1}{\sqrt{2}}, \sqrt{2}\right]$. Nevertheless, we do not know if this definition provide the existence of a unit mesh for $\left(I_{3}(\mathbf{x})\right)_{\mathbf{x} \in \mathbb{R}^{3}}$. In the following, this question of existence is studied by means of the space filling tetrahedra.

Non-regular space filling tetrahedra. The study of space filling tetrahedra is an old geometrical question $[33,37]$. In the past, it has been demonstrated that there exist sets of non-regular space filling tetrahedra: the Sommerville tetrahedra [38] and the Goldberg tetrahedra family [21].

The Sommerville tetrahedra are based on particular splittings of the unit cube, see Figure 2.4. We recall these tetrahedra thanks to their vertices coordinates, only the last vertex distinguishes them. $K$ is denoted $\left(\mathbf{x}_{\mathbf{1}}, \mathbf{x}_{\mathbf{2}}, \mathbf{x}_{\mathbf{3}}, \mathbf{x}_{\mathbf{4}}\right)$ with $\mathbf{x}_{\mathbf{1}}=(0,0,0)$, $\mathbf{x}_{\mathbf{2}}=\left(\frac{1}{2},-\frac{1}{2}, \frac{1}{2}\right), \mathbf{x}_{\mathbf{3}}=\left(\frac{1}{2}, \frac{1}{2}, \frac{1}{2}\right)$ and

- $\mathbf{x}_{\mathbf{4}}=\left(\frac{1}{2}, 0,0\right)$ for the Sommeville tetrahedron 1

- $\mathbf{x}_{\mathbf{4}}=(1,0,0)$ for the Sommeville tetrahedron 2

- $\mathbf{x}_{\mathbf{4}}=\left(\frac{1}{2},-\frac{1}{2},-\frac{1}{2}\right)$ for the Sommeville tetrahedron 3

- $\mathbf{x}_{\mathbf{4}}=\left(\frac{1}{2}, 0,-\frac{1}{4}\right)$ for the Sommeville tetrahedron 4 .
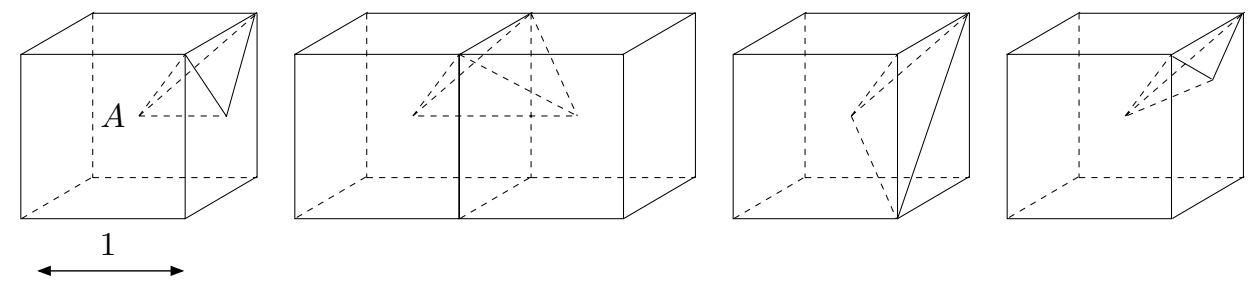

FIG. 2.4. From left to right, the Sommerville tetrahedra 1, 2, 3 and 4.

The Goldberg tetrahedra are based on the splitting of a prism the basis of which is the equilateral triangle. Their coordinates depend on an initial choice of two lengths $a$ and $e$. We specify one of the Goldberg tetrahedra for the specific choice $a=\frac{1}{3}$ and $e=1$ :

$$
\text { - } \mathbf{x}_{\mathbf{1}}=(0,0,0), \mathbf{x}_{\mathbf{2}}=(0,0,1), \mathbf{x}_{\mathbf{3}}=\left(0,1, \frac{1}{3}\right) \text { and } \mathbf{x}_{\mathbf{4}}=\left(\frac{\sqrt{3}}{2}, \frac{1}{2}, \frac{2}{3}\right) \text {. }
$$

We propose now to compare these space filling tetrahedra to the unit regular tetrahedron. To this end, all these tetrahedra are scaled such that their volumes are equal to $\frac{\sqrt{2}}{12}$. The resulting lengths of edges for each tetrahedron are specified in Table 2.1. We notice that the proposed notion of quasi-unit element is only verified for the Sommerville tetrahedra 1 and 2, and the Goldberg tetrahedron. Therefore, there exists space filling tetrahedra that are quasi-units for the metric $I_{3}$ in the sense proposed above.

Now, the case of a constant anisotropic metric $\mathcal{M}$ is studied. We consider the pattern around a vertex, i.e., the vertex ball, composed only with the second Sommerville tetrahedron. This pattern exists as it fills $\left(\mathbb{R}^{3}, \mathcal{M}\right)$. The vertex ball is mapped back in 
the natural Euclidean space thanks to the application $\mathcal{M}^{\frac{1}{2}}$. Then, we notice that the non-regularity of the second Sommerville tetrahedron leads necessarily to the creation (in the Euclidean space) of several different anisotropic tetrahedra. However, all these different tetrahedra have the same length of edges and the same volume in the metric $\mathcal{M}$. Consequently, filling space with only one tetrahedra is possible for all isotropic metrics of the form $\alpha I_{3}$, but a set of tetrahedra is required to fill the Euclidean space anisotropically.

Controlling the volume. Unfortunately, the weaker constraint on the length of edges can lead to the generation of quasi-unit elements with a null volume. For instance in $\left(\mathbb{R}^{3}, I_{3}\right)$, the regular tetrahedron with length of edges equal to $\sqrt{2}$ is quasiunit for $I_{3}$. However, if one of its vertex is projected orthogonally on the opposite face, then a quasi-unit element of null volume is obtained. Indeed, three edges are of length $\sqrt{2}$ and the three other are of length $\frac{\sqrt{3}}{6} \approx 0.816 \in\left[\frac{1}{\sqrt{2}}, \sqrt{2}\right]$. In consequence, controlling only the length of the edges is not sufficient, the volume must also be controlled to relax the notion of unit element. Practically, this is achieved by controlling the ratio between the sum of the square length of the edges over the volume of the element. All quantities are computed within the prescribed metric. With this ratio, we define a function $Q_{\mathcal{M}}$ that measures the quality in $\mathcal{M}$ of an element $K$ :

$$
Q_{\mathcal{M}}(K)=\frac{36}{3^{\frac{1}{3}}} \frac{|K|_{\mathcal{M}}^{\frac{2}{3}}}{\sum_{i=1}^{6} \ell_{\mathcal{M}}^{2}\left(\mathbf{e}_{\mathbf{i}}\right)} \in[0,1] .
$$

The constant $\frac{36}{3^{\frac{1}{3}}}$ is chosen so that $Q_{\mathcal{M}}$ is equal to 1 for the equilateral tetrahedron whatever the length of its edges. For a null volume tetrahedron, $Q_{\mathcal{M}}$ is 0 . The qualities of the space filling tetrahedra are given in Table 2.1. Notice that $Q_{\mathcal{M}}$ only quantifies the gap to the shape of the regular tetrahedron. We deduce the following definition of quasi-unit element, which is also practically used by mesh generators:

Definition 2.7 (Quasi-unit element). A tetrahedron $K$ defined by its list of edges $\left(\mathbf{e}_{i}\right)_{i=1,6}$ is said quasi-unit for $\mathcal{M}$ if

$$
\forall i \in[1,6], \quad \ell_{\mathcal{M}}\left(\mathbf{e}_{\mathbf{i}}\right) \in\left[\frac{1}{\sqrt{2}}, \sqrt{2}\right] \quad \text { and } \quad Q_{\mathcal{M}}(K) \in[\alpha, 1] \quad \text { with } \quad \alpha>0 .
$$

In our case, $\alpha=0.8$ is an acceptable value as it enables the Sommerville tetrahedra 1 and 2, and the Goldberg tetrahedron to be generated.

REMARK 1. Instead of considering $Q_{\mathcal{M}}$, the quality function $\frac{1}{Q_{\mathcal{M}}}$ can be considered. As the variation range becomes $[1, \infty[$, the discrimination of bad elements is made easier.

2.4. Continuous terminology. Propositions 2.2 and 2.4 highlight a duality between discrete entities and continuous ones. It results that, in the proposed continuous framework, a metric tensor $\mathcal{M}$ is assimilated to a continuous element and a continuous mesh of a domain $\Omega$ is defined by a collection of continuous elements $\mathbf{M}=(\mathcal{M}(\mathbf{x}))_{\mathbf{x} \in \Omega}$, i.e., a Riemannian metric space. In particular, for an element, this duality is justified by strict analogy between discrete and continuous notions: orientation vs. matrix $\mathcal{R}$, stretching vs. $r_{i}$ and size vs. $d$. For a mesh, we point out the duality between the number of vertices and $\mathcal{C}(\mathbf{M})$. Proposition 2.3 also illustrates a 
duality between geometric quantities. This duality will be even reinforced in the next section by studying the interpolation error.

In what follows, the continuous terminology is employed to emphasize the exhibited duality. In particular, a continuous element $\mathcal{M}$ is a metric tensor and a continuous mesh of a domain $\Omega \subset \mathbb{R}^{3}$ is a Riemannian metric space $\mathbf{M}=(\mathcal{M}(\mathbf{x}))_{\mathbf{x} \in \boldsymbol{\Omega}}$.

3. Continuous linear interpolation error: discrete-continuous duality. In the previous section, a continuous framework has been introduced to model elements and meshes. Now, we aim at applying this framework in the context of error estimation. However, as our intent is to propose a fully discrete-continuous duality, it is not enough to derive only the optimal mesh arising from an interpolation error bound as in classical studies on interpolation error $[6,17,24]$. Instead, we want to evaluate the interpolation error for any functions on any continuous meshes without imposing some optimality conditions as alignment, equi-distribution, ...

Let $(\mathcal{M}(\mathbf{x}))_{\mathbf{x} \in \Omega}$ be a continuous mesh of a domain $\Omega$ and let $u$ be a non linear function which is assumed to be only twice continuously differentiable. We seek a wellposed definition of the continuous linear interpolation error $\left\|u-\pi_{\mathcal{M}} u\right\|_{\mathbf{L}^{1}(\Omega)}$ related to a continuous mesh $(\mathcal{M}(\mathbf{x}))_{\mathbf{x} \in \Omega}$ which implies a well-posed definition of a linear continuous interpolate $\pi_{\mathcal{M}} u$. More precisely, we would like the continuous linear interpolation error to be a reliable mathematical model of $\left\|u-\Pi_{h} u\right\|_{\mathbf{L}^{1}\left(\Omega_{h}\right)}$ where $\Pi_{h}$ is defined by a mesh $\mathcal{H}$ of a discretized domain $\Omega_{h}$ which is a unit mesh with respect to $(\mathcal{M}(\mathbf{x}))_{\mathbf{x} \in \Omega}$. In fine, this means that considering $\left\|u-\pi_{\mathcal{M}} u\right\|_{\mathbf{L}^{1}(\Omega)}$ is equivalent to consider $\left\|u-\Pi_{h} u\right\|_{\mathbf{L}^{1}\left(\Omega_{h}\right)}$.

The error analysis is first done locally, i.e, in a tangent space of $(\mathcal{M}(\mathbf{x}))_{\mathbf{x} \in \Omega}$ at a given point $\mathbf{a}$. In the tangent space, $(\mathcal{M}(\mathbf{x}))_{\mathbf{x} \in \Omega}$ reduces to the continuous element $\mathcal{M}(\mathbf{a})$. In other words, the analysis is performed locally at the element level. The function $u$ is approximated by its local quadratic taylor expansion. Indeed, terms of order greater than two can be neglected while studying the linear interpolation error. Then, a global error estimate is derived by taking into account the variation of the continuous mesh and of the function.

3.1. Interpolation error in $\mathbf{L}^{1}$ norm for quadratic functions. In this section, we consider a quadratic function $u$ defined on a domain $\Omega \subset \mathbb{R}^{3}$. The function is given by its matrix representation:

$$
\forall \mathbf{x} \in \Omega, \quad u(\mathbf{x})=\frac{1}{2}{ }^{t} \mathbf{x} H \mathbf{x},
$$

\begin{tabular}{|c|c|c|c|c|c|c|c|c|}
\hline Tetrahedron & Coeff. & \multicolumn{6}{|c|}{ Length of edges } & Quality \\
\hline Sommerville 1 & $\overline{\overline{\sqrt{2}}}$ & 0.70 & $\overline{1.22}$ & 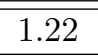 & $\overline{1.0}$ & 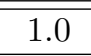 & 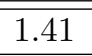 & 0.800 \\
\hline Sommerville 2 & $2^{\frac{1}{6}}$ & 1.12 & 0.970 & 0.970 & 0.970 & 0.970 & 1.12 & 0.954 \\
\hline Sommerville 3 & $2^{\frac{1}{6}}$ & 0.970 & 0.970 & 0.970 & 1.12 & 1.59 & 1.12 & 0.763 \\
\hline Sommerville 4 & $12^{-\frac{1}{3}} 2^{\frac{3}{2}}$ & 0.691 & 1.07 & 1.07 & 1.12 & 1.12 & 1.23 & 0.886 \\
\hline Goldberg & $3^{-\frac{1}{2}} 2^{\frac{1}{6}}$ & 0.932 & 0.990 & 1.12 & 1.12 & 0.990 & 0.990 & 0.950 \\
\hline
\end{tabular}

TABLE 2.1

Space filling tetrahedra characteristics. Coeff. is the coefficient that scales the tetrahedron onto a unit volume tetrahedron, i.e., $|K|=\sqrt{2} / 12$. The length of edges and the tetrahedron quality $Q_{I_{3}}$ given by Formula (2.5) are provided. 
where $H$ is a symmetric matrix representing the Hessian of $u$. For every symmetric matrix $H,|H|$ denotes the positive symmetric matrix deduced from $H$ by taking the absolute values of its eigenvalues. The function $u$ is linearly interpolated on a tetrahedron $K$ defined by its vertices list: $K=\left(\mathbf{x}_{1}, \mathbf{x}_{2}, \mathbf{x}_{3}, \mathbf{x}_{4}\right) .|K|$ denotes the Euclidean volume of $K$. We denote by $\Pi_{h} u$ the linear interpolate of $u$ on $K$. We can now state the following result:

Proposition 3.1. For every quadratic function $u$, its linear interpolation error in $\mathbf{L}^{1}$ norm on a tetrahedron $K$ verifies:

$$
\left\|u-\Pi_{h} u\right\|_{\mathbf{L}^{1}(K)} \leq \frac{|K|}{40} \sum_{i=1}^{6}{ }^{t} \mathbf{e}_{i}|H| \mathbf{e}_{i}
$$

where $\left(\mathbf{e}_{i}\right)_{i=1,6}$ is the set of edges of $K$.

The previous inequality becomes an equality when $u$ is elliptic or parabolic.

Proof. The proof consists in deriving an exact error estimate of the point-wise interpolation error within the element $K: e(\mathbf{x})=\left(u-\Pi_{h} u\right)(\mathbf{x})$ for $\mathbf{x} \in K$. This error is then integrated over $K$. To derive $e$, we use the standard reference element technique. Reference element $K_{r e f}$ is defined by its four vertices coordinates:

$$
\widehat{\mathbf{x}}_{1}={ }^{t}(0,0,0), \widehat{\mathbf{x}}_{2}={ }^{t}(1,0,0), \widehat{\mathbf{x}}_{3}={ }^{t}(0,1,0) \text { and } \widehat{\mathbf{x}}_{4}={ }^{t}(0,0,1) .
$$

All the computations are done on $K_{\text {ref }}$ and the result is then mapped onto the current element $K$ by using the following affine mapping:

$$
\mathbf{x}=\mathbf{v}_{1}+B_{K} \widehat{\mathbf{x}} \text { with } B_{K}=\left[\mathbf{x}_{2}-\mathbf{x}_{1}, \mathbf{x}_{3}-\mathbf{x}_{1}, \mathbf{x}_{4}-\mathbf{x}_{1}\right], \quad \mathbf{x} \in K, \quad \hat{\mathbf{x}} \in K_{r e f} .
$$

The matrix $B_{k}$ is given as a function of the following edges:

$$
\mathbf{e}_{1}=\mathbf{x}_{2}-\mathbf{x}_{1}, \quad \mathbf{e}_{2}=\mathbf{x}_{3}-\mathbf{x}_{1} \text { and } \mathbf{e}_{3}=\mathbf{x}_{4}-\mathbf{x}_{1},
$$

so that $B_{K}=\left[\mathbf{e}_{1}, \mathbf{e}_{2}, \mathbf{e}_{3}\right]$. The quadratic function $u$ reads in the frame of $K_{r e f}$ :

$$
u(\mathbf{x}(\widehat{\mathbf{x}}))=\frac{1}{2}{ }^{t} \mathbf{x}_{1} H \mathbf{x}_{1}+\frac{1}{2}{ }^{t} \mathbf{x}_{1} H B_{K} \widehat{\mathbf{x}}+\frac{1}{2}{ }^{t} \widehat{\mathbf{x}}^{t} B_{K} H \mathbf{x}_{1}+\frac{1}{2}{ }^{t} \widehat{\mathbf{x}}^{t} B_{K} H B_{K} \widehat{\mathbf{x}} .
$$

As we consider the linear interpolation, linear and constant terms of $u(\mathbf{x}(\widehat{\mathbf{x}}))$ are exactly interpolated. Without loss of generality, these terms are neglected and only quadratic terms are kept. Indeed, if we consider $\tilde{u}(\mathbf{x})=\frac{1}{2}{ }^{t} \widehat{\mathbf{x}}^{t} B_{K} H B_{K} \widehat{\mathbf{x}}$, then it comes:

$$
e(\mathbf{x})=\left(u-\Pi_{h} u\right)(\mathbf{x})=\left(\tilde{u}-\Pi_{h} \tilde{u}\right)(\mathbf{x}) .
$$

We can now consider $\tilde{u}$ instead of $u$. However for the sake of clarity, we keep on writing $u$ and not $\tilde{u}$. We rewrite $u$ in a matrix form:

$$
u(\mathbf{x}(\widehat{\mathbf{x}}))=\frac{1}{2}{ }^{t}\left(\begin{array}{c}
\hat{x} \\
\hat{y} \\
\hat{z}
\end{array}\right)\left[\begin{array}{ccc}
{ }^{t} \mathbf{e}_{1} H \mathbf{e}_{1} & { }^{t} \mathbf{e}_{1} H \mathbf{e}_{2} & { }^{t} \mathbf{e}_{1} H \mathbf{e}_{3} \\
{ }^{t} \mathbf{e}_{2} H \mathbf{e}_{1} & { }^{t} \mathbf{e}_{2} H \mathbf{e}_{2} & { }^{t} \mathbf{e}_{2} H \mathbf{e}_{3} \\
{ }^{t} \mathbf{e}_{3} H \mathbf{e}_{1} & { }^{t} \mathbf{e}_{3} H \mathbf{e}_{2} & { }^{t} \mathbf{e}_{3} H \mathbf{e}_{3}
\end{array}\right]\left(\begin{array}{c}
\hat{x} \\
\hat{y} \\
\hat{z}
\end{array}\right) .
$$

$u$ in $K_{\text {ref }}$ reads:

$$
u(\mathbf{x}(\widehat{\mathbf{x}}))=\frac{1}{2}\left[\begin{array}{cccc}
\left({ }^{t} \mathbf{e}_{1} H \mathbf{e}_{1}\right) \hat{x}^{2} & +\left({ }^{t} \mathbf{e}_{2} H \mathbf{e}_{2}\right) \hat{y}^{2} & +\left({ }^{t} \mathbf{e}_{3} H \mathbf{e}_{3}\right) \hat{z}^{2} & + \\
2\left({ }^{t} \mathbf{e}_{1} H \mathbf{e}_{2}\right) \hat{x} \hat{y} & +2\left({ }^{t} \mathbf{e}_{1} H \mathbf{e}_{3}\right) \hat{x} \hat{z} & + & 2\left({ }^{t} \mathbf{e}_{2} H \mathbf{e}_{3}\right) \hat{y} \hat{z}
\end{array}\right] .
$$


$u$ is now linearly interpolated on $K_{\text {ref }}$. Its linear interpolate $\Pi_{h} u(\widehat{\mathbf{x}})$ writes $a \widehat{x}+b \widehat{y}+$ $c \widehat{z}+d$, where coefficients $(a, b, c, d) \in \mathbb{R}^{4}$ satisfies the following linear system ensuring the $\mathcal{P}_{1}$ exactness, i.e., $\Pi_{h} u\left(\mathbf{v}_{i}\right)=u\left(\mathbf{v}_{i}\right)$ for all $i \in[1,4]$ :

$$
\left\{\begin{array}{l}
\Pi_{h} u\left(\mathbf{v}_{1}\right)=d=u(\mathbf{x}((0,0,0))=0, \\
\Pi_{h} u\left(\mathbf{v}_{2}\right)=a=u\left(\mathbf{x}((1,0,0))=\frac{1}{2}\left({ }^{t} \mathbf{e}_{1} H \mathbf{e}_{1}\right),\right. \\
\Pi_{h} u\left(\mathbf{v}_{3}\right)=b=u\left(\mathbf{x}((0,1,0))=\frac{1}{2}\left({ }^{t} \mathbf{e}_{2} H \mathbf{e}_{2}\right),\right. \\
\Pi_{h} u\left(\mathbf{v}_{4}\right)=c=u\left(\mathbf{x}((0,0,1))=\frac{1}{2}\left({ }^{t} \mathbf{e}_{3} H \mathbf{e}_{3}\right) .\right.
\end{array}\right.
$$

The solution of the previous linear system gives the final expression of $\Pi_{h} u$ :

$$
\Pi_{h} u(\mathbf{x}(\widehat{\mathbf{x}}))=\frac{1}{2}\left[\left({ }^{t} \mathbf{e}_{1} H \mathbf{e}_{1}\right) \hat{x}+\left({ }^{t} \mathbf{e}_{2} H \mathbf{e}_{2}\right) \hat{y}+\left({ }^{t} \mathbf{e}_{3} H \mathbf{e}_{3}\right) \hat{z}\right] .
$$

The exact point-wise interpolation error $e(\mathbf{x})$ is then given by:

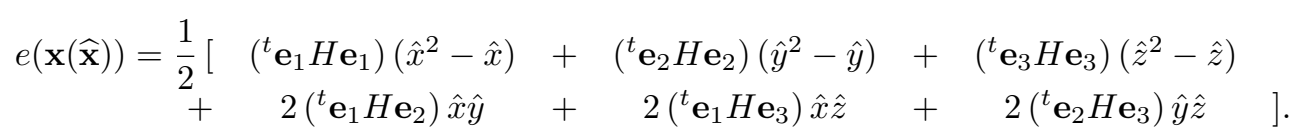

From this equality, estimate in $\mathbf{L}^{1}, \mathbf{L}^{2}$ or $\mathbf{H}^{1}$ can be deduced by considering the change of variables given by the mapping $B_{K}$. Indeed, for every function $F$, its integration over $K$ can be computed through its expression in $K_{\text {ref }}$ :

$$
\int_{K} F(\mathbf{x}) \mathrm{d} x \mathrm{~d} y \mathrm{~d} z=\int_{K_{r e f}} F(\mathbf{x}(\widehat{\mathbf{x}}))\left|\operatorname{det}\left(B_{K}\right)\right| \mathrm{d} \hat{x} \mathrm{~d} \hat{y} \mathrm{~d} \hat{z},
$$

As $6|K|=\operatorname{det}\left(B_{k}\right)$, previous equality becomes:

$$
\int_{K} F(\mathbf{x}) \mathrm{d} x \mathrm{~d} y \mathrm{~d} z=6|K| \int_{K_{r e f}} F(\mathbf{x}(\widehat{\mathbf{x}})) \mathrm{d} \hat{x} \mathrm{~d} \hat{y} \mathrm{~d} \hat{z} .
$$

Consequently, the interpolation error in $\mathbf{L}^{1}$ norm is evaluated by a direct integration of $|e(\mathbf{x})|$. When $u$ is concave or convex, we have: $\left|\left(u-\Pi_{h} u\right)(\mathbf{x})\right|=\left(u-\Pi_{h} u\right)(\mathbf{x})$ in the convex case and $\left|\left(u-\Pi_{h} u\right)(\mathbf{x})\right|=-\left(u-\Pi_{h} u\right)(\mathbf{x})$ in the concave case. The error reads:

$$
\begin{aligned}
\left\|u-\Pi_{h} u\right\|_{\mathbf{L}^{1}(K)}=\frac{|K|}{40} \mid & 2\left({ }^{t} \mathbf{e}_{1} H \mathbf{e}_{2}+{ }^{t} \mathbf{e}_{1} H \mathbf{e}_{3}+{ }^{t} \mathbf{e}_{2} H \mathbf{e}_{3}\right) \\
& -3\left({ }^{t} \mathbf{e}_{1} H \mathbf{e}_{1}+{ }^{t} \mathbf{e}_{2} H \mathbf{e}_{2}+{ }^{t} \mathbf{e}_{3} H \mathbf{e}_{3}\right) \mid .
\end{aligned}
$$

Using the conventions of Figure 2.2, the crossed terms can be expressed only in terms of $\mathbf{e}_{i} H \mathbf{e}_{i}$ for $i=1, \ldots, 6$ :

$$
\left\{\begin{array}{c}
2{ }^{t} \mathbf{e}_{1} H \mathbf{e}_{2}={ }^{t} \mathbf{e}_{1} H \mathbf{e}_{1}+{ }^{t} \mathbf{e}_{2} H \mathbf{e}_{2}-{ }^{t} \mathbf{e}_{4} H \mathbf{e}_{4}, \\
2{ }^{t} \mathbf{e}_{1} H \mathbf{e}_{3}={ }^{t} \mathbf{e}_{1} H \mathbf{e}_{1}+{ }^{t} \mathbf{e}_{3} H \mathbf{e}_{3}-{ }^{t} \mathbf{e}_{5} H \mathbf{e}_{5}, \\
{ }^{t}{ }^{t} \mathbf{e}_{2} H \mathbf{e}_{3}={ }^{t} \mathbf{e}_{2} H \mathbf{e}_{2}+{ }^{t} \mathbf{e}_{3} H \mathbf{e}_{3}-{ }^{t} \mathbf{e}_{6} H \mathbf{e}_{6} .
\end{array}\right.
$$

We deduce:

$$
\left|2\left({ }^{t} \mathbf{e}_{1} H \mathbf{e}_{2}+{ }^{t} \mathbf{e}_{1} H \mathbf{e}_{3}+{ }^{t} \mathbf{e}_{2} H \mathbf{e}_{3}\right)-3\left({ }^{t} \mathbf{e}_{1} H \mathbf{e}_{1}+{ }^{t} \mathbf{e}_{2} H \mathbf{e}_{2}+{ }^{t} \mathbf{e}_{3} H \mathbf{e}_{3}\right)\right|=\left|\sum_{i=1}^{6}{ }^{t} \mathbf{e}_{i} H \mathbf{e}_{i}\right| .
$$


If $u$ is hyperbolic, the inequality $\frac{1}{2}|\mathbf{x} H \mathbf{x}| \leq \frac{1}{2} \mathbf{x}|H| \mathbf{x}$ is used to conclude the proof in the general case.

The same proof applies in 2D. For a quadratic function $u$, the linear interpolation error on a triangle $K$ is given by:

$$
\left\|u-\Pi_{h} u\right\|_{\mathbf{L}^{1}(K)} \leq \frac{|K|}{24} \sum_{i=1}^{3}{ }^{t} \mathbf{e}_{i}|H| \mathbf{e}_{i} .
$$

Error estimates in $\mathbf{L}^{2}$ norm and in $\mathbf{H}^{1}$ norm can also be derived. We refer to [4, 32] and references therein for their evaluations. These error estimates are classically used to exhibit mesh quality functions and to obtain the best element shape minimizing the interpolation error [1]. In the continuous mesh framework, the interpolation error estimate in $\mathbf{L}^{1}$ norm is used to prove some exactness properties of the continuous linear interpolate.

REMARK 2 (Safety principle). Even if it is possible to define exactly the linear interpolation error in $\mathbf{L}^{1}$ norm for hyperbolic functions, we do not consider these expressions from a practical point of view. We prefer to consider $|H|$ instead of $H$ transforming the function into an elliptic or a parabolic one. It comes to in overestimating the error for hyperbolic functions. Indeed, it seems that we do not take any advantages of considering the null error directions for mesh adaptation.

3.2. Linear interpolation on a continuous element. Let $\mathcal{M}$ be a continuous element and $u$ be a quadratic positive function (see Remark 2). We study the interpolation error for the class of all unit discrete elements with respect to $\mathcal{M}$, given by Definition 2.1. Figure 2.1 depicts for a given metric tensor $\mathcal{M}$ some unit elements. We can now state the main result:

TheOREM 3.2. For all unit elements $K$ with respect to $\mathcal{M}$, the interpolation error of $u$ in $\mathbf{L}^{1}$ norm does not depend on the element shape and is only a function of the Hessian $H$ of $u$ and of continuous element $\mathcal{M}$.

- In $3 D$, for all unit elements $K$ for $\mathcal{M}$, the following equality holds:

$$
\left\|u-\Pi_{h} u\right\|_{\mathbf{L}^{1}(K)}=\frac{\sqrt{2}}{240} \operatorname{det}\left(\mathcal{M}^{-\frac{1}{2}}\right) \operatorname{trace}\left(\mathcal{M}^{-\frac{1}{2}} H \mathcal{M}^{-\frac{1}{2}}\right) .
$$

- In $2 D$, for all unit elements $K$ for $\mathcal{M}$, the following equality holds:

$$
\left\|u-\Pi_{h} u\right\|_{\mathbf{L}^{1}(K)}=\frac{\sqrt{3}}{64} \operatorname{det}\left(\mathcal{M}^{-\frac{1}{2}}\right) \operatorname{trace}\left(\mathcal{M}^{-\frac{1}{2}} H \mathcal{M}^{-\frac{1}{2}}\right) .
$$

Proof. According to Proposition 3.1, the interpolation error in $\mathbf{L}^{1}$ norm of a quadratic positive function $u$ on an element $K$ is:

$$
\left\|u-\Pi_{h} u\right\|_{\mathbf{L}^{1}(K)}=\frac{|K|}{40} \sum_{i=1}^{6}{ }^{t} \mathbf{e}_{i} H \mathbf{e}_{i} .
$$

Then, if $K$ is unit with respect to $\mathcal{M}$, the previous interpolation error is expressed by:

$$
\left\|u-\Pi_{h} u\right\|_{\mathbf{L}^{1}(K)}=\frac{\sqrt{2}}{240} \operatorname{det}\left(\mathcal{M}^{-\frac{1}{2}}\right) \operatorname{trace}\left(\mathcal{M}^{-\frac{1}{2}} H \mathcal{M}^{-\frac{1}{2}}\right)
$$


thanks to the geometric invariants related to the volume, Relation (2.2), and to the square lengths of the edges, Relation (2.3).

We note the strong analogy with classical interpolation error estimate for Lagrange interpolation [7]:

- The term $\operatorname{det}\left(\mathcal{M}^{-\frac{1}{2}}\right)$ stands for the Jacobian of the affine transformation from the reference element $\hat{K}$ onto the current element $K$. In our continuous framework, it is the Jacobian of the affine mapping between the reference continuous element unit ball $\mathcal{B}_{I_{3}}$ onto the current continuous element unit ball $\mathcal{B}_{\mathcal{M}}$.

- The term trace $\left(\mathcal{M}^{-\frac{1}{2}} H \mathcal{M}^{-\frac{1}{2}}\right)$ stands for the semi-norm involved in classical error estimates. Generally, this semi-norm contains the anisotropic behavior of the estimate. In the continuous framework, the trace-term gives the alignment correlation between the principal directions of the Hessian $H$ and the principal directions of the metric $\mathcal{M}$.

Relation (3.1) shows that the infinite set of discrete elements that are unit for a given continuous element $\mathcal{M}$ achieves the same interpolation error, and moreover, shows that this interpolation error is only expressed with continuous quantities: the continuous element $\mathcal{M}$ and the Hessian of the function $u$. Consequently, Theorem 3.2 points out that the metric alone contains enough information to describe completely the linear interpolation error in $\mathbf{L}^{1}$ norm. In other words, this theorem confirms that the use of metric-based mesh adaptation is particularly well suited to control anisotropically the interpolation error. In the past, this efficiency has been observed practically on real life problems, see for instance $[5,14,15,17,30,34,36,40]$.

3.3. Continuous linear interpolate. The main difficulty in defining the continuous linear interpolate is to connect a discrete error computed on an element to a local continuous error that is defined point-wise. Indeed, the discrete interpolation error in norm $\mathbf{L}^{1}$ is integrated on the element $K$. On the contrary, a continuous mesh is a function $\mathbf{x} \mapsto \mathcal{M}(\mathbf{x})$ defined at each point $\mathbf{x}$ of $\Omega$.

Suppose now that the continuous mesh $(\mathcal{M}(\mathbf{x}))_{\mathbf{x} \in \Omega}$ is varying and that the function $u$ is no more quadratic but only twice continuously differentiable. If Equality (3.1) of Theorem 3.2 does not hold anymore, all the terms of the right-hand-side $\mathcal{M}$ and $H$ are well defined continuously. The definition of a continuous interpolate follows up from this consideration.

We denote by $u_{Q}$ the quadratic approximation of a smooth function $u$. At point a, $u_{Q}$ is defined in the vicinity of $\mathbf{a}$ as the truncated second order Taylor expansion of $u$ :

$$
\forall \mathbf{x} \in \mathcal{V}(\mathbf{a}), \quad u_{Q}(\mathbf{a} ; \mathbf{x})=u(\mathbf{a})+\nabla u(\mathbf{a})(\mathbf{x}-\mathbf{a})+\frac{1}{2}\langle(\mathbf{x}-\mathbf{a}), H(\mathbf{a})(\mathbf{x}-\mathbf{a})\rangle .
$$

When no confusion is possible, the notation $u_{Q}(\mathbf{a} ; \mathbf{x})$ is replaced by $u_{Q}(\mathbf{x}) . u_{Q}$ is a complete quadratic form composed of a constant term, a linear term and finally a quadratic term.

The first result of this section provides an equivalence formula between discrete and continuous views locally around a point $\mathbf{a}$ of the domain. In the vicinity of $\mathbf{a}, u_{Q}$ approximates $u$ and $(\mathcal{M}(\mathbf{x}))_{\mathbf{x} \in \Omega}$ reduces to $\mathcal{M}(\mathbf{a})$ in the tangent space. We can now state the main result:

THEOREM 3.3 (Discrete-continuous equivalence). Let $u$ be a twice continuously differentiable fonction of a domain $\Omega$ and $(\mathcal{M}(\mathbf{x}))_{\mathbf{x} \in \Omega}$ be a continuous mesh of $\Omega$. 
Then, there exists a unique continuous linear interpolate function $\pi_{\mathcal{M}}$ such that:

$$
\forall \mathbf{a} \in \Omega, \quad\left|u-\pi_{\mathcal{M}} u\right|(\mathbf{a})=2 \frac{\left\|u_{Q}-\Pi_{h} u_{Q}\right\|_{\mathbf{L}^{1}(K)}}{|K|},
$$

for every $K$ unit element with respect to $\mathcal{M}(\mathbf{a})$.

The proof is given hereafter, we first look at the consequences of this theorem.

COROLlary 3.4. Let u be a twice continuously differentiable fonction of a domain $\Omega$ and $(\mathcal{M}(\mathbf{x}))_{\mathbf{x} \in \Omega}$ be a continuous mesh of $\Omega$. Then, the following continuous linear interpolation estimate holds in $3 D$ :

$$
\begin{aligned}
\forall \mathbf{a} \in \Omega, \quad\left|u-\pi_{\mathcal{M}} u\right|(\mathbf{a}) & =\frac{1}{10} \operatorname{trace}\left(\mathcal{M}(\mathbf{a})^{-\frac{1}{2}}|H(\mathbf{a})| \mathcal{M}(\mathbf{a})^{-\frac{1}{2}}\right) \\
& =\frac{1}{10}\left(d(\mathbf{a})^{-\frac{2}{3}} \sum_{i=1}^{3} r_{i}(\mathbf{a})^{\frac{2}{3}}{ }^{t} \mathbf{v}_{i}(\mathbf{a})|H(\mathbf{a})| \mathbf{v}_{i}(\mathbf{a})\right)
\end{aligned}
$$

In $2 D$, the estimate is:

$$
\begin{aligned}
\forall \mathbf{a} \in \Omega, \quad\left|u-\pi_{\mathcal{M}} u\right|(\mathbf{a}) & =\frac{1}{8} \operatorname{trace}\left(\mathcal{M}(\mathbf{a})^{-\frac{1}{2}}|H(\mathbf{a})| \mathcal{M}(\mathbf{a})^{-\frac{1}{2}}\right) \\
& =\frac{1}{8}\left(d(\mathbf{a})^{-1} \sum_{i=1}^{2} r_{i}(\mathbf{a})^{t} \mathbf{v}_{i}(\mathbf{a})|H(\mathbf{a})| \mathbf{v}_{i}(\mathbf{a})\right) .
\end{aligned}
$$

Proof. In 3D, for all unit elements $K$ with respect to $\mathcal{M}(\mathbf{a})$, the error estimation (3.1) can be rewritten as follow for the quadratic function $u_{Q}$ approximating $u$ in the vicinity of $\mathbf{a}$ :

$$
\frac{\left\|u_{Q}-\Pi_{h} u_{Q}\right\|_{\mathbf{L}^{1}(K)}}{|K|}=\frac{1}{20} \operatorname{trace}\left(\mathcal{M}(\mathbf{a})^{-\frac{1}{2}}|H(\mathbf{a})| \mathcal{M}(\mathbf{a})^{-\frac{1}{2}}\right) .
$$

Then, expressing $\mathcal{M}(\mathbf{a})$ as a function of the continuous mesh parameters given by the decomposition of Proposition 2.4 leads to:

$$
\frac{\left\|u_{Q}-\Pi_{h} u_{Q}\right\|_{\mathbf{L}^{1}(K)}}{|K|}=\frac{1}{20}\left(d(\mathbf{a})^{-\frac{2}{3}} \sum_{i=1}^{3} r_{i}(\mathbf{a})^{\frac{2}{3}}{ }^{t} \mathbf{v}_{i}(\mathbf{a})|H(\mathbf{a})| \mathbf{v}_{i}(\mathbf{a})\right)
$$

where the $\left(\mathbf{v}_{i}(a)\right)_{i=1,3}$ stand for the eigenvectors of $\mathcal{M}(\mathbf{a})$.

This result shows that the continuous point-wise linear interpolation can be decomposed into the product of two terms:

- a first term that control the accuracy, this density term is directly connected to the size of the continuous element,

- a second term that measures alignment deviation between the continuous element orientation and the anisotropy features of the function $u$.

REMARK 3 (Convergence order). Note that if the first term (involving the density) is rewritten in term of metric size accordingly to Proposition 2.4, $d(\mathbf{a})^{\frac{2}{3}}$ in $3 D$ and $d(\mathbf{a})^{-1}$ in $2 D$ are both of the order of $h^{-2}(\mathbf{a})$. This shows the typical second order of convergence for the linear interpolation error. We study the convergence analysis within the continuous framework in Part II [29].

It is possible to give a geometric interpretation of this estimate. This interpretation illustrates the impact of a continuous mesh on the error iso-values and, 
consequently, gives some clue toward the control of the error by means of a continuous mesh. The term $\mathcal{M}^{-\frac{1}{2}}(\mathbf{a})|H(\mathbf{a})| \mathcal{M}^{-\frac{1}{2}}$ (a) corresponds to the frame change related to the continuous mesh local orientation. Given a symmetric matrix $|H(\mathbf{a})|$, the corresponding quadratic form is: $f=\frac{1}{2}^{t} \mathbf{x}|H(\mathbf{a})| \mathbf{x}$.

The matrix $\mathcal{M}^{-\frac{1}{2}}(\mathbf{a})|H(\mathbf{a})| \mathcal{M}^{-\frac{1}{2}}$ (a) corresponds to a new quadratic form observed in the space deformed by $\mathcal{M}(\mathbf{a})$. Indeed, if we consider the change of coordinates $\tilde{\mathbf{x}}=\mathcal{M}^{\frac{1}{2}}(\mathbf{a}) \mathbf{x}$, we define a new quadratic form $\tilde{f}$ :

$$
\begin{aligned}
\tilde{f}(\tilde{\mathbf{x}})={ }^{t} \mathbf{x}|H(\mathbf{a})| \mathbf{x} & ={ }^{t}\left(\mathcal{M}^{-\frac{1}{2}}(\mathbf{a}) \tilde{\mathbf{x}}\right)|H(\mathbf{a})| \mathcal{M}^{-\frac{1}{2}}(\mathbf{a}) \tilde{\mathbf{x}} \\
& ={ }^{t} \tilde{\mathbf{x}}\left(\mathcal{M}^{-\frac{1}{2}}(\mathbf{a})|H(\mathbf{a})| \mathcal{M}^{-\frac{1}{2}}(\mathbf{a})\right) \tilde{\mathbf{x}}
\end{aligned}
$$

Iso-values of $\tilde{f}$ and $f$ are different when they are seen in the canonical Euclidean space. In fact, viewing a quadratic form for different continuous meshes changes its iso-values in the Euclidean space, i.e., the real physical space. It is then possible to control the error iso-values by modifying $\mathcal{M}$. This is the main principle of mesh adaptation, but here formulated in a continuous framework. Mesh adaptation principle: Classical metric-based mesh adaptation consists in finding a metric field that provides isotropic iso-values of the error function, see pionneer work [6].

These results demonstrate that both the interpolation error and the linear interpolate $\Pi_{h}$ have continuous counterparts. It is then a step forward in finding a complete duality between the discrete and the continuous views. From a practical point of view, we deduce the following analogy. Given a unit mesh $\mathcal{H}$ of a domain $\Omega_{h}$ with respect to a continuous mesh $(\mathcal{M}(\mathbf{x}))_{\mathbf{x} \in \Omega}$, the global interpolation error is:

$$
\left\|u-\Pi_{h} u\right\|_{\mathbf{L}^{1}\left(\Omega_{h}\right)}=\sum_{K \in \mathcal{H}}\left\|u-\Pi_{h} u\right\|_{\mathbf{L}^{1}(K)} .
$$

In the continuous case, the discrete summation becomes an integral:

$$
\left\|u-\pi_{\mathcal{M}} u\right\|_{\mathbf{L}^{1}(\Omega)}=\int_{\Omega}\left|u-\pi_{\mathcal{M}} u\right|(\mathbf{x}) \mathrm{d} \mathbf{x} .
$$

Note that there is no global guarantee on the continuous interpolation error reliability given by Relation (3.3). For instance, there is no a priori relationship between (3.2) and (3.3). The only guarantee is the local equivalence given by Theorem 3.3. However, the local guarantee becomes global when the mesh is unit with respect to a constant metric tensor (this does not necessary implied that the mesh is uniform) and when the function is quadratic. In this specific case, by neglecting error due to the boundary discretization, we have the equality:

$$
2\left\|u-\Pi_{h} u\right\|_{\mathbf{L}^{1}\left(\Omega_{h}\right)}=\left\|u-\pi_{\mathcal{M}} u\right\|_{\mathbf{L}^{1}(\Omega)},
$$

for all unit meshes $\mathcal{H}$ with respect to $(\mathcal{M}(\mathbf{x}))_{\mathbf{x} \in \Omega}$. The numerical examples in Part II [29] will numerically demonstrate the efficiency of the continuous model. In particular, we will observe that:

- the model is accurate and the equivalence $(3.2) \approx(3.3)$ is observed for non quadratic functions and non-constant continuous meshes,

- the error due to the fact that mesh generator generates edges with length not stricly equal to one is negligible. In particular, the range for the lengths of the edges given in Definition 2.7 ensures reliable numerical results. 
The constant 2 involved in Theorem 3.3 arises in the perfect case where the mesh is only composed of perfect unit elements. Note this is only possible in $2 \mathrm{D}$ whereas it is always false in 3D due the impossibility to tessel the space only with the regular tetrahedron. In a more practical situation, this constant needs to be evaluated from one unit mesh in order to estimate the deviation between the continuous mesh complexity with respect to the number of vertices of the unit mesh. In other words, the number of vertices $N_{v}$ of a unit mesh verifies the following function:

$$
N_{v}=C N
$$

where $N$ is the continuous mesh complexity and $C$ a constant. The constant $C$ depends on the domain shape, the mesh generator used and the unit mesh resulting quality. Consequently, it reflects how far the current mesh is from the perfect unity. Several examples are given in the numerical examples section and illustrates this relation.

To conclude this section, the proof of Theorem 3.3 is given. This proof is based on the exact expression of the continuous linear interpolate:

Proposition 3.5. The continuous interpolate $\pi_{\mathcal{M} u}$ evaluated at $\mathbf{a} \in \Omega$ for a continuous mesh $(\mathcal{M}(\mathbf{x}))_{\mathbf{x} \in \Omega}$ and for a smooth function $u$ is given by:

$$
\pi_{\mathcal{M}} u(\mathbf{a})=p^{*}(0),
$$

where $p^{*}$ is the unique linear polynomial solution of:

$$
p^{*}=\min _{p \in \mathcal{P}^{1}}\left\|u_{Q}-p\right\|_{\mathbf{L}^{2}\left(\mathcal{B}_{\mathcal{M}}\right)},
$$

where $u_{Q}$ is the quadratic model of $u$ at $\mathbf{a}$ and $\mathcal{B}_{\mathcal{M}}$ is the unit ball of $\mathcal{M}$ at $\mathbf{a} . \pi_{\mathcal{M}}$ is given by

$$
\pi_{\mathcal{M}} u(\mathbf{a})=u(\mathbf{a})+\nabla u(\mathbf{a})+\frac{1}{c_{n}} \operatorname{trace}\left(\mathcal{M}^{-\frac{1}{2}}(\mathbf{a}) H(\mathbf{a}) \mathcal{M}^{-\frac{1}{2}}(\mathbf{a})\right),
$$

where $c_{n}$ is a constant that depends only on the space dimension:

$$
c_{2}=\frac{1}{8} \quad \text { and } \quad c_{3}=\frac{1}{10} .
$$

Proof. The quadratic model $u_{Q}$ of $u$ at point a defined by:

$$
u_{Q}(\mathbf{a} ; \mathbf{x})=u(\mathbf{a})+\nabla u(\mathbf{a})(\mathbf{x}-\mathbf{a})+\frac{1}{2}\langle(\mathbf{x}-\mathbf{a}), H(\mathbf{a})(\mathbf{x}-\mathbf{a})\rangle,
$$

becomes after the translation $\mathbf{x} \mapsto \mathbf{x}+\mathbf{a}$

$$
u_{Q}(\mathbf{x})=\frac{1}{2}{ }^{t} \mathbf{x} H(\mathbf{a}) \mathbf{x}+\nabla u(\mathbf{a}) \mathbf{x}+u(\mathbf{a})
$$

The linear polynomial $p^{*}$ is given by:

$$
p^{*}: \mathbf{x} \in \mathcal{B}_{\mathcal{M}} \mapsto{ }^{t} \mathbf{g} \mathbf{x}+c,
$$

where $c \in \mathbb{R}$ and $\mathbf{g} \in \mathbb{R}^{3}$. As the space of linear polynomials $\mathcal{P}^{1}$ and $\mathcal{B}_{\mathcal{M}}$ are convex, $p^{*}$ exists and is unique. We seek for $p^{*}$ verifying the following condition:

$$
\forall p \in \mathcal{P}^{1}, \int_{\mathcal{B}_{\mathcal{M}}}\left(u_{Q}(\mathbf{x})-p^{*}(\mathbf{x})\right) p(\mathbf{x}) \mathrm{d} \mathbf{x}=0 .
$$


In particular, it is true for the following basis of $\mathcal{P}^{1}: \mathbf{x} \mapsto 1, \mathbf{x} \mapsto x_{i}$, for $i \in\{1,2,3\}$. The previous condition leads to:

$$
\int_{\mathcal{B}_{\mathcal{M}}}\left(u_{Q}(\mathbf{x})-p^{*}(\mathbf{x})\right) \mathrm{d} \mathbf{x}=0 \text { and } \int_{\mathcal{B}_{\mathcal{M}}}\left(u_{Q}(\mathbf{x})-p^{*}(\mathbf{x})\right) x_{i} \mathrm{~d} \mathbf{x}=0,
$$

for $i=\{1,2,3\}$. The initial integration domain $\mathcal{B}_{\mathcal{M}}$ is mapped onto $\mathcal{B}_{I_{3}}$ by using the following one-to-one change of variables:

$$
\begin{aligned}
& \mathcal{B}_{\mathcal{M}} \longrightarrow \mathcal{B}_{I_{3}} \\
& \mathbf{x} \quad \longmapsto \mathbf{y}=\mathcal{M}(\mathbf{a})^{\frac{1}{2}} \mathbf{x} .
\end{aligned}
$$

Functions $u_{Q}$ and $p^{*}$ becomes:

$$
\begin{aligned}
& u_{Q}(\mathbf{x})=\tilde{u}_{Q}(\mathbf{y})=\frac{1}{2}^{t} \mathbf{y} \mathcal{M}(\mathbf{a})^{-\frac{1}{2}} H(\mathbf{a}) \mathcal{M}(\mathbf{a})^{-\frac{1}{2}} \mathbf{y}+{ }^{t} \mathbf{y} \mathcal{M}(\mathbf{a})^{-\frac{1}{2}} \nabla u(\mathbf{a})+u(\mathbf{a}), \\
& p^{*}(\mathbf{x})=\tilde{p}^{*}(\mathbf{y})={ }^{t} \mathbf{y} \mathcal{M}(\mathbf{a})^{-\frac{1}{2}} \mathbf{g}+c .
\end{aligned}
$$

We now consider the following basis: $\mathbf{y} \mapsto 1, \mathbf{y} \mapsto y_{i}$, for $i \in\{1,2,3\}$. Equations (3.4) become:

$$
\begin{aligned}
& \int_{\mathcal{B}_{I_{3}}}\left(\tilde{u}_{Q}(\mathbf{y})-\tilde{p}^{*}(\mathbf{y})\right) \operatorname{det}\left(\mathcal{M}(\mathbf{a})^{-\frac{1}{2}}\right) \mathrm{d} \mathbf{y}=0, \\
& \int_{\mathcal{B}_{I_{3}}}\left(\tilde{u}_{Q}(\mathbf{y})-\tilde{p}^{*}(\mathbf{y})\right) y_{i} \operatorname{det}\left(\mathcal{M}(\mathbf{a})^{-\frac{1}{2}}\right) \mathrm{d} \mathbf{y}=0,
\end{aligned}
$$

for $i=\{1,2,3\}$. A formal integration gives in 3D [39]:

$$
\begin{aligned}
& \left(\frac{2}{15} \operatorname{trace}\left(\mathcal{M}(\mathbf{a})^{-\frac{1}{2}} H(\mathbf{a}) \mathcal{M}(\mathbf{a})^{-\frac{1}{2}}\right)+\frac{4}{3}(u(\mathbf{a})-c)\right) \operatorname{det}\left(\mathcal{M}(\mathbf{a})^{-\frac{1}{2}}\right)=0, \\
& \left(\frac{4}{15} \mathcal{M}(\mathbf{a})^{-\frac{1}{2}}(\nabla u(\mathbf{a})-\mathbf{g})\right) \operatorname{det}\left(\mathcal{M}(\mathbf{a})^{-\frac{1}{2}}\right)=0,
\end{aligned}
$$

from which the expression of $p^{*}$ is deduced:

$$
\mathbf{g}=\nabla u(\mathbf{a}) \text { and } c=u(\mathbf{a})+\frac{1}{10} \operatorname{trace}\left(\mathcal{M}(\mathbf{a})^{-\frac{1}{2}} H(\mathbf{a}) \mathcal{M}(\mathbf{a})^{-\frac{1}{2}}\right) .
$$

Finally, for the $2 D$ case, the expression of $p^{*}$ is given by:

$$
\mathbf{g}=\nabla u(\mathbf{a}) \text { and } c=u(\mathbf{a})+\frac{1}{8} \operatorname{trace}\left(\mathcal{M}(\mathbf{a})^{-\frac{1}{2}} H(\mathbf{a}) \mathcal{M}(\mathbf{a})^{-\frac{1}{2}}\right) .
$$

The proof of Theorem 3.3 is deduced from the definition of $\pi_{\mathcal{M}} u(\mathbf{a})$.

In the case where the continuous mesh is constant and the function $u$ quadratic, we verify $(3.2)=(3.3)$.

Notice that using the $\mathbf{L}^{2}$ projection of the quadratic model $u_{Q}$ of $u$ is necessary to ensure the specific equivalence between the discrete linear interpolate $\Pi_{h}$ and the continuous linear interpolate $\pi_{\mathcal{M}}$ of Theorem 3.3. However, the continuous linear interpolate is still well defined if we use the function $u$ instead of $u_{Q}$. It seems then possible to define the continuous linear interpolate for far less regular functions. For instance, one may consider only functions that are locally $\mathbf{L}^{2}$. An open problem is then to find a discrete linear interpolation operator which enables discrete and discontinuous approaches to be linked. Some works involving other interpolation operators have already been considered, we can cite the developments in [16] that derive interpolation error estimate based on the Clément's interpolate [8]. 
4. Conclusion. In this paper, we have proposed a continuous framework to model a mesh and its elements. The model is based of the notion of Riemannian metric space. In this context, the following analogy has been proved. Geometric invariants have been exhibited that connect a metric tensor to the set of all the discrete elements which are represented by this metric. Metric tensors have then been used to continuously model discrete elements. As the behavior of a Riemannian metric space is obtained by patching together the behavior of each of its tangent spaces, which are defined by metric spaces, the global continuous mesh model arises from gathering together all the continuous elements. This continuous mesh model is emphasized by rewriting the symmetric definite positive matrix representing the metric tensor at each point of the computational domain. Continuous mesh features as density, orientation and stretching ratio are pointed out. The discrete projection is then based on the notion of unit mesh. A unit mesh with respect to a continuous mesh is provided by the use of an adaptive mesh generator. Consequently, the discrete image depends on the method used to generate the mesh and is not unique.

The discrete-continuous duality has been completed by providing a continuous interpolation error estimate and a well-posed definition of the continuous linear interpolate. The latter is based on an exact relation connecting the discrete error to the continuous one. This main result states that the linear interpolation error on the class of unit elements is only a function of the associated continuous element and of the considered function. In other words, the notion of continuous mesh handles sufficient information to completely model the interpolation error which is a priori only discrete.

\section{REFERENCES}

[1] R. E. BAnK And R. K. Smith, Mesh smoothing using a posteriori error estimates, SIAM J. Numer. Anal., 34 (1997), pp. 979-997.

[2] M. Berger, Geometry 1 \& 2 2, Springer Verlag, Berlin, 1987.

[3] — A panoramic view of Riemannian geometry, Springer Verlag, Berlin, 2003.

[4] M. Berzins, Solution-based mesh quality for triangular and tetrahedral meshes, in Proc. of 6th Meshing Rountable, 1997, pp. 427-436.

[5] C. Bottasso, Anisotropic mesh adaption by metric-driven optimization, Int. J. Numer. Meth. Engrg., 60 (2004), pp. 597-639.

[6] M. J. Castro-Díaz, F. Hecht, B. Mohammadi, and O. Pironneau, Anisotropic unstructured mesh adaptation for flow simulations, Int. J. Numer. Meth. Fluids, 25 (1997), pp. 475-491.

[7] P. Ciarlet, The Finite Element Method for Elliptic Problems, North-Holland, Amsterdam, 1978.

[8] P. Clément, Approximation by finite element functions using local regularization, Revue Française d'Automatique, Informatique et Recherche Opérationnelle, R-2 (1975), pp. 7784.

[9] G. Compère, J.-F. Remacle, J. Jansson, and J. Hoffman, A mesh adaptation framework for dealing with large deforming meshes, Int. J. Numer. Meth. Engrg., 82 (2010), pp. 843-867.

[10] T. Coupez, Génération de maillages et adaptation de maillage par optimisation locale, Revue Européenne des Éléments Finis, 9 (2000), pp. 403-423.

[11] F. Courty, D. Leservoisier, P. George, And A. Dervieux, Continuous metrics and mesh adaptation, Appl. Numer. Math., 56 (2006), pp. 117-145.

[12] M. do Carmo, Differential geometry of curves and surfaces, Prentice Hall, 1976.

[13] C. Dobrzynski and P. Frey, Anisotropic Delaunay mesh adaptation for unsteady simulations, in Proc. of 17th Meshing Rountable, Springer, 2008, pp. 177-194.

[14] J. Dompierre, M. Vallet, M. Fortin, Y. Bourgault, and W. Habashi, Anisotropic mesh adaptation: towards a solver and user independent CFD, in AIAA 35th Aerospace Sciences Meeting and Exhibit, AIAA-1997-0861, Reno, NV, USA, Jan 1997.

[15] L. Formaggia, S. Micheletti, and S. Perotto, Anisotropic mesh adaptation in computational fluid dynamics: Application to the advection-diffusion-reaction and the Stokes 
problems, Appl. Numer. Math., 51 (2004), pp. 511-533.

[16] L. Formaggia And S. Perotto, New anisotropic a prioiri error estimate, Numer. Math., 89 (2001), pp. 641-667.

[17] P. Frey and F. Alauzet, Anisotropic mesh adaptation for CFD computations, Comput. Meth. Appl. Mech. Engrg., 194 (2005), pp. 5068-5082.

[18] P. Frey and P. George, Mesh generation. Application to finite elements, ISTE Ltd and John Wiley \& Sons, 2nd ed., 2008

[19] P. J. FREY, Yams, a fully automatic adaptive isotropic surface remeshing procedure, RT-0252, INRIA, nov 2001.

[20] P.-L. George, Gamanic3d, adaptive anisotropic tetrahedral mesh generator, Technical Note, INRIA, 2003.

[21] M. Goldberg, Three infinite families of tetrahedral space-fillers, J. Comb. Theory, Ser. A, 16 (1974), pp. 348-354.

[22] F. Hecht, BAMG: bidimensional anisotropic mesh generator, available from http://wwwrocq.inria.fr/gamma/cdrom/www/bamg/eng.htm, INRIA-Rocquencourt, France, 1998.

[23] F. Hecht And B. Mohammadi, Mesh adaptation by metric control for multi-scale phenomena and turbulence, in 35th AIAA Aerospace Sciences Meeting and Exhibit, AIAA-1997-0859, Reno, NV, USA, Jan 1997.

[24] W. Huang, Metric tensors for anisotropic mesh generation, J. Comp. Phys., 204 (2005), pp. 633-665.

[25] W. Jones, E. Nielsen, And M. Park, Validation of 3D adjoint based error estimation and mesh adaptation for sonic boom reduction, in 44th AIAA Aerospace Sciences Meeting and Exhibit, AIAA-2006-1150, Reno, NV, USA, Jan 2006.

[26] P. LAUg and H. Bourochaki, BL2D-V2, mailleur bidimensionnel adaptatif, RT-0275, INRIA, 2003.

[27] X. Li, M. S. Shephard, And M. W. Beall, 3D anisotropic mesh adaptation by mesh modification, Comput. Meth. Appl. Mech. Engrg., 194 (2005), pp. 4915-4950.

[28] A. Loseille, Adaptation de maillage 3D anisotrope multi-échelles et ciblé à une fonctionnelle. Application à la prédiction haute-fidélité du bang sonique., $\mathrm{PhD}$ thesis, Université Pierre et Marie Curie, Paris VI, Paris, France, 2008.

[29] A. Loseille and F. Alauzet, Continuous mesh framework. Part II: validations and applications, SIAM J. Numer. Anal., (2010). Submitted.

[30] A. Loseille, A. Dervieux, P. Frey, And F. Alauzet, Achievement of global second-order mesh convergence for discontinuous flows with adapted unstructured meshes, in 37th AIAA Fluid Dynamics Conference and Exhibit, AIAA-2007-4186, Miami, FL, USA, Jun 2007.

[31] A. Loseille And R. LöHner, Anisotropic adaptive simulations in aerodynamics, AIAA Paper, AIAA-10-0169 (2010).

[32] E. J. NADLER, Piecewise linear approximation on triangulations of a planar regions, PhD thesis, Brown university, Providence, 1985.

[33] D. J. NAYLOR, Filling space with tetrahedra, Int. J. Numer. Meth. Engrg., 44 (1999), pp. 13831395.

[34] C. C. Pain, A. P. Umpleby, C. R. E. de Oliveira, and A. J. H. Goddard, Tetrahedral mesh optimisation and adaptivity for steady-state and transient finite element calculations, Comput. Meth. Appl. Mech. Engrg., 190 (2001), pp. 3771-3796.

[35] M. PICASSO, An anisotropic error indicator based on Zienkiewicz-Zhu error estimator: Application to elliptic and parabolic problems, SIAM J. Sci. Comput., 24 (2003), pp. 1328-1355.

[36] E. Schall, D. Leservoisier, A. Dervieux, And B. Koobus, Mesh adaptation as a tool for certified computational aerodynamics, Int. J. Numer. Meth. Fluids, 45 (2004), pp. 179-196.

[37] M. Senechal, Which tetrahedra fill space?, Mathematics Magazine, 54 (1981), pp. 227-243.

[38] D. Y. M. Sommerville, Space-filling tetrahedra in euclidean space, in Proc. Edinburgh Math. Soc., vol. 41, 1923, pp. 49-57.

[39] S. Sykora, Quantum theory and the bayesian inference problems, J. Statistical Phys., 11 (1974), pp. 17-27.

[40] A. Tam, D. Ait-Ali-Yahia, M. P. Robichaud, M. Moore, V. Kozel, and W. G. Habashi, Anisotropic mesh adaptation for $3 D$ flows on structured and unstructured grids, Comput. Meth. Appl. Mech. Engrg., 189 (2000), pp. 1205-1230.

[41] M.-G. Vallet, Génération de maillages éléments finis anisotropes et adaptatifs, PhD thesis, Université Pierre et Marie Curie, Paris VI, Paris, France, 1992. 